ARTICLE

https://doi.org/10.1038/s41467-020-14293-1

\title{
YAP/TAZ direct commitment and maturation of lymph node fibroblastic reticular cells
}

Sung Yong Choi ${ }^{1,2,11}$, Hosung Bae (i) ${ }^{3,11}$, Sun-Hye Jeong 4,11, Intae Park1, Hyunsoo Cho ${ }^{1}$, Seon Pyo Hong ${ }^{1}$, Da-Hye Lee ${ }^{4}$, Choong-kun Lee ${ }^{1,2}$, Jin-Sung Park1, Sang Heon Suh¹,2, Jeongwoon Choi ${ }^{1,3}$, Myung Jin Yang 1,3, Jeon Yeob Jang ${ }^{5}$, Lucas Onder ${ }^{6}$, Jeong Hwan Moon ${ }^{7}$, Han-Sin Jeong ${ }^{8}$, Ralf H. Adams (10) ${ }^{9}$, Jin-Man Kim ${ }^{10}$, Burkhard Ludewig (D) ${ }^{6}$, Joo-Hye Song (1) ${ }^{1}$, Dae-Sik Lim ${ }^{4 \star} \&$ Gou Young Koh (1) 1,2,3*

Fibroblastic reticular cells (FRCS) are immunologically specialized myofibroblasts of lymphoid organ, and FRC maturation is essential for structural and functional properties of lymph nodes (LNs). Here we show that YAP and TAZ (YAP/TAZ), the final effectors of Hippo signaling, regulate FRC commitment and maturation. Selective depletion of YAP/TAZ in FRCs impairs FRC growth and differentiation and compromises the structural organization of LNs, whereas hyperactivation of YAP/TAZ enhances myofibroblastic characteristics of FRCs and aggravates $L N$ fibrosis. Mechanistically, the interaction between YAP/TAZ and p52 promotes chemokine expression that is required for commitment of FRC lineage prior to lymphotoxin- $\beta$ receptor (LT $\beta R$ ) engagement, whereas $L T \beta R$ activation suppresses YAP/TAZ activity for FRC maturation. Our findings thus present YAP/TAZ as critical regulators of commitment and maturation of FRCs, and hold promise for better understanding of FRC-mediated pathophysiologic processes.

\footnotetext{
${ }^{1}$ Center for Vascular Research, Institute for Basic Science (IBS), Daejeon 34141, Republic of Korea. ${ }^{2}$ Graduate School of Medical Science and Engineering, Korea Advanced Institute of Science and Technology (KAIST), Daejeon 34141, Republic of Korea. ${ }^{3}$ Biomedical Science and Engineering Interdisciplinary Program, KAIST, Daejeon 34141, Republic of Korea. ${ }^{4}$ Department of Biological Science, KAIST, Daejeon 34141, Republic of Korea. ${ }^{5}$ Department of Otorhinolaryngology, Ajou University School of Medicine, Suwon 16499, Republic of Korea. ${ }^{6}$ Institute of Immunobiology, Kantossipital St. Gallen, St. Gallen 9007, Switzerland. ${ }^{7}$ Department of Otorhinolaryngology - Head and Neck Surgery, Dankook University College of Medicine, Cheonan 31116, Republic of Korea. ${ }^{8}$ Department of Otorhinolaryngology - Head and Neck Surgery, Samsung Medical Center, Sungkyunkwan University School of Medicine, Seoul 06351, Republic of Korea. ${ }^{9}$ Department of Tissue Morphogenesis, Max Planck Institute for Molecular Biomedicine, and Faculty of Medicine, University of Münster, Münster MD-48149, Germany. ${ }^{10}$ Department of Pathology, Chungnam National University School of Medicine, Daejeon 35015, Republic of Korea. ${ }^{11}$ These authors contributed equally: Sung Yong Choi, Hosung Bae, Sun-Hye Jeong. *email: daesiklim@kaist.ac.kr; gykoh@kaist.ac.kr
} 
A fine network of fibroblastic reticular cells (FRCs) is essential for maintaining lymph node (LN) structure and function ${ }^{1-3}$. FRCs regulate immune cell entry into the LN via high endothelial venules (HEVs) and confer compartmentalization of lymphocytes within the LN by secretion of essential chemokines ${ }^{1,4-6}$. In this regard, fibrotic damage to FRCs by chronic inflammation and cancer has been shown to seriously deteriorate systemic immune responses by $\mathrm{FRCs}^{7-10}$. Thus, proper differentiation of FRC progenitors into mature FRCs during development is critical for acquisition of immunoregulatory characters and initiation of adaptive immune response and chemokine production ${ }^{1,11-16}$

The differentiation of FRCs involves differentiation of a poorly defined population of mesenchymal cells into FRC precursors, which further develop into mature FRCs ${ }^{1,12}$. Whereas the molecular details involved in the latter process such as lymphotoxin- $\beta$ receptor (LT $\beta R$ ) and receptor activation of NF-kB ligand (RANKL)-mediated interactions of lymphoid tissue inducer (LTi) cells with FRC precursors have been thoroughly described ${ }^{17,18}$, characterization of stromal cells and signaling pathways involved in the commitment steps of FRC development are incompletely defined $^{19-21}$.

The core of the Hippo pathway consists of large tumor suppressors 1 and 2 (LATS1/2), and yes-associated protein (YAP) and transcriptional co-activator with PDZ-binding motif (TAZ), which are final effectors of the Hippo pathway that exert their functions by mainly interacting with the TEAD/TEF family of transcription factors 22,23 . Upon activation of Hippo pathway, LATS1/2 become phosphorylated and inhibit the activities of YAP and TAZ (YAP/TAZ). This pathway acts as a key regulator of cellular proliferation, differentiation, organ size control, tissue homeostasis, and regeneration ${ }^{22,24-26}$. Despite such diverse and important roles of Hippo pathway in a wide range of biological processes, its role is largely unexplored in LN FRCs.

We hypothesize that the Hippo pathway plays crucial roles in regulating differentiation of FRCs, with relevance to structural and functional properties of LNs. Using FRC-specific genetic targeting and lineage-tracing approaches, we show that YAP/TAZ deficiency impairs FRC differentiation, while hyperactivation of YAZ/TAZ induces myofibroblastic FRCs and LN fibrosis. Thus, we present YAP/TAZ as critical regulators in maintaining FRC integrity and hold promise for better understanding of FRCmediated physiologic and pathologic conditions.

\section{Results}

YAP/TAZ support growth and structure of LN by FRCs. To gain insights into the role of the Hippo pathway in LNs, we first examined the expressions and distributions of YAP/TAZ in human and mouse LNs. Both YAP/TAZ were enriched in $\alpha$ smooth muscle actin (aSMA) ${ }^{+}$FRCs of healthy human LNs (Supplementary Fig. 1a). Similarly, YAP/TAZ were highly and equally distributed in nucleus and cytoplasm of FRCs, in addition to endothelial cells of high endothelial venules (HEVs) in mouse LNs (Supplementary Fig. 1b).

To elucidate the roles of YAP/TAZ in LN FRCs during development, we generated $\mathrm{Yap} / \mathrm{Ta} \mathrm{z}^{\mathrm{ARRC}}$ mice by crossing Ccl19Cre mouse ${ }^{20}$ and $Y a p^{\text {flox/flox } 27} /$ Taz $^{\text {flox/flox } 28}$ mouse and analyzed them at 8 weeks after birth (Fig. 1a and Supplementary Fig. 1c). Cre-negative but flox/flox-positive mice among the littermates were defined as wild-type (WT) mice for each experiment. Although we confirmed the depletion of YAP/TAZ in FRCs of Yap/Taz ${ }^{\Delta \mathrm{FRC}}$ mice, there was no difference in body growth except for a slight reduction in LN weight (Fig. 1b-d). Of note, LNs of $\mathrm{Yap} / \mathrm{Taz} \mathrm{z}^{\mathrm{FRC}}$ mice revealed reduced total cell number $(\sim 64.9 \%)$, decreased proportion $(\sim 42.9 \%)$ and number $(\sim 61.1 \%)$ of FRCs among $\mathrm{CD}^{-} 5^{-}$stromal cells, which were less proliferative $(\sim 65.2 \%)$ but had no difference in apoptosis (Fig. $1 \mathrm{~d}-\mathrm{g}$ and Supplementary Fig. 1d-f). However, LNs of Yap/Taz ${ }^{\triangle \mathrm{FRC}}$ mice contained similar numbers of BECs and LECs compared with WT (Fig. 1g). To address the selective role of YAP or TAZ in FRCs, we compared LNs of $Y a p^{\Delta \mathrm{FRC}}$ or $T a z^{\Delta \mathrm{FRC}}$ mice with those of WT and Yap/Taz ${ }^{\Delta \mathrm{FRC}}$ mice (Supplemental Fig. 2a). Of note, decreased LN weight and reduced cellularity observed in $\mathrm{Yap} / \mathrm{Taz}^{\Delta \mathrm{FRC}}$ mice were not evident in $\mathrm{Yap}^{\Delta \mathrm{FRC}}$ or $\mathrm{Taz}{ }^{\Delta \mathrm{FRC}}$ mice (Supplemental Fig. 2b), suggesting largely redundant roles of YAP and TAZ in FRCs.

Further analysis of LNs revealed that the distinct border between $\mathrm{B}$ and $\mathrm{T}$ cell zones was disrupted in Yap/Taz ${ }^{\Delta \mathrm{FRC}}$ mice, but not in $Y_{a p}{ }^{\Delta \mathrm{FRC}}$ or Taz ${ }^{\Delta \mathrm{FRC}}$ mice (Fig. 1h and Supplementary Fig. 2c). However, neither proliferation nor apoptosis of immune cells was altered (Supplementary Fig. 3a,b). Instead, analysis of isolated FRCs revealed that mRNA levels of lymphoid chemokines for immune cell trafficking were significantly attenuated in Yap/Taz ${ }^{\triangle \mathrm{FRC}}$ mice compared with WT (Fig. 1i and Supplementary Fig. 3c). Indeed, injection of labeled cells showed that the recruitment of transferred $\mathrm{GFP}^{+} \mathrm{T}$ cells $(\sim 63.5 \%)$ and DsRed ${ }^{+} \mathrm{B}$ cells $(-57.0 \%)$ were significantly impaired in LNs of Yap/Taz ${ }^{\Delta \mathrm{FRC}}$ mice compared with WT mice (Fig. 1j, k).

Next, we assessed whether the antiviral immune responses would be affected as a consequence of the changes in $\mathrm{Yap} / \mathrm{Taz}^{\Delta \mathrm{FRC}}$ mice by intranasal inoculation of influenza virus $(\mathrm{A} / \mathrm{PR} / 8 / 34)$. Although there were no apparent differences in immune cell composition in bone marrow, thymus, peripheral circulating blood, and LNs (Supplementary Fig. 4a-i), $\mathrm{Yap} / \mathrm{Taz}^{\triangle \mathrm{FRC}}$ mice had less activated interferon- $\gamma$ (IFN- $\gamma$ )secreting effector $\mathrm{CD}^{+} \mathrm{T}$ cells, and were severely affected compared with WT mice after challenge with influenza virus (Fig. 1l,m). However, no significant differences were observed in $\mathrm{IL}^{2}{ }^{+} \mathrm{CD}^{+}$helper $\mathrm{T}$ cells and anti-A/PR/8 IgG antibody production between WT and Yap/Taz ${ }^{\Delta \mathrm{FRC}}$ mice (Supplementary Fig. 5a-c). To better characterize the changes in the FRC phenotype upon Yap/Taz deletion, we generated WT ${ }^{\text {FRC-TR }}$ and Yap/Taz ${ }^{\triangle \mathrm{FRC}-\mathrm{TR}}$ mice by crossing Yap/Taz ${ }^{\Delta \mathrm{FRC}}$ mouse with Rosa26-tdTomato reporter mouse (Supplementary Fig. 6a,b). LNs of Yap/Taz $\triangle \mathrm{FRC}-\mathrm{TR}$ mice revealed increased FRC surface area $(\sim 1.5$-fold $)$ and ER-TR7 ${ }^{+}$naked conduits $(\sim 5.2$-fold) compared with WT ${ }^{\text {FRC-TR }}$ (Supplementary Fig. 6c,d). In concordance, electron microscope revealed that $\sim 45.8 \%$ of conduits were not covered with FRCs and $\sim 30.4 \%$ of collagen fibrils were irregularly distributed in the remaining conduits in LNs of Yap/Taz ${ }^{\Delta \mathrm{FRC}}$ mice compared with WT (Supplementary Fig. 6e,f). However, conduit integrity and functionality were relatively preserved despite the reduced FRC coverage in Yap/Taz ${ }^{\mathrm{FRC}}$ mice compared with WT (Supplementary Fig. 6g,h), suggesting that minor structural defects in LN conduit system are unlikely the main cause of altered immune cell trafficking.

YAP/TAZ hyperactivation impairs differentiation of FRCs. To specifically hyperactivate YAP/TAZ in FRCs during LN development, we generated Lats $1 / 2^{\Delta \mathrm{FRC}}$ mutants by crossing Ccl19-Cre mouse and Lats $1^{\text {flox/flox } 29 / L a t s 2 \text { flox/flox } 30}$ mouse (Fig. 2a). Neonatal Lats $1 / 2^{\Delta \mathrm{FRC}}$ mice exhibited substantial growth retardation with lethality at 16-21 days after birth (Supplementary Fig. 7a-c). Moreover, they showed severely disrupted structural organization of LNs with impaired lymphatic drainage (Fig. $2 \mathrm{~b}$ and Supplementary Fig. $7 \mathrm{~d}, \mathrm{e})$. LN weight $(\sim 61 \%)$, total number of cells within the LN ( 98\%), and the fraction of stromal cell populations of LNs were also markedly reduced in Lats $1 / 2^{\triangle \mathrm{FRC}}$ mice compared with $\mathrm{WT}$ (Fig. 2c and Supplementary Fig. 7f). 
a

$$
\begin{aligned}
& \square \text { Yap/Taz }{ }^{\text {floxfllox }}: \text { WT } \\
& \square \text { Cc/19-Cre x Yap/Taz } \\
& \text { floxflox }: \text { Yap/Taz }{ }^{\Delta F R C}
\end{aligned}
$$

b

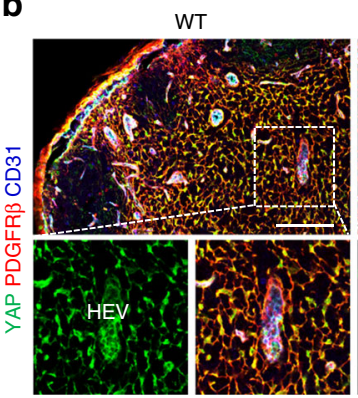

C
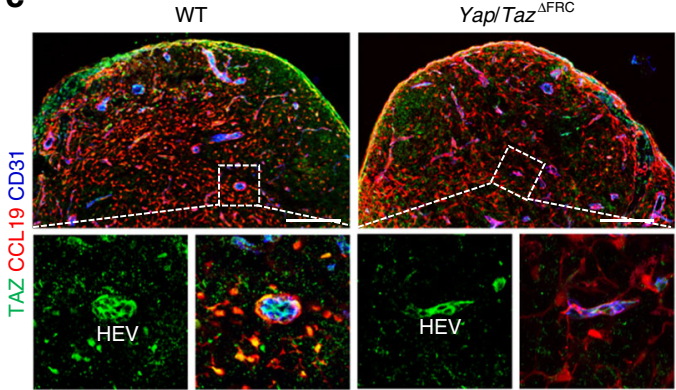

d
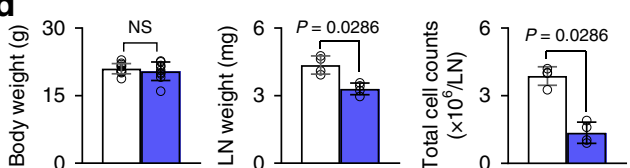

e
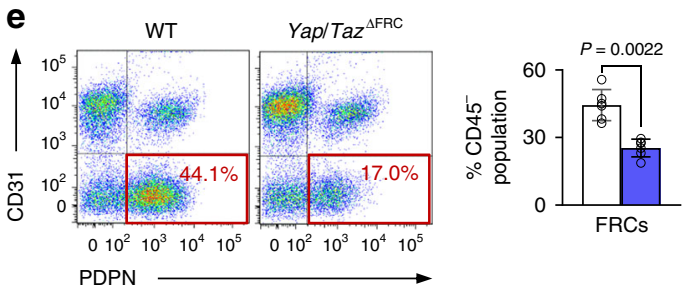

$\mathbf{f}$

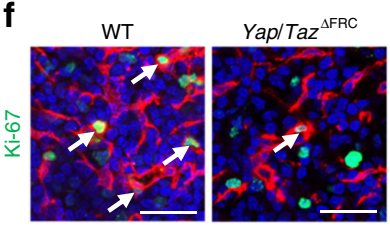

PDGFRB DAPI
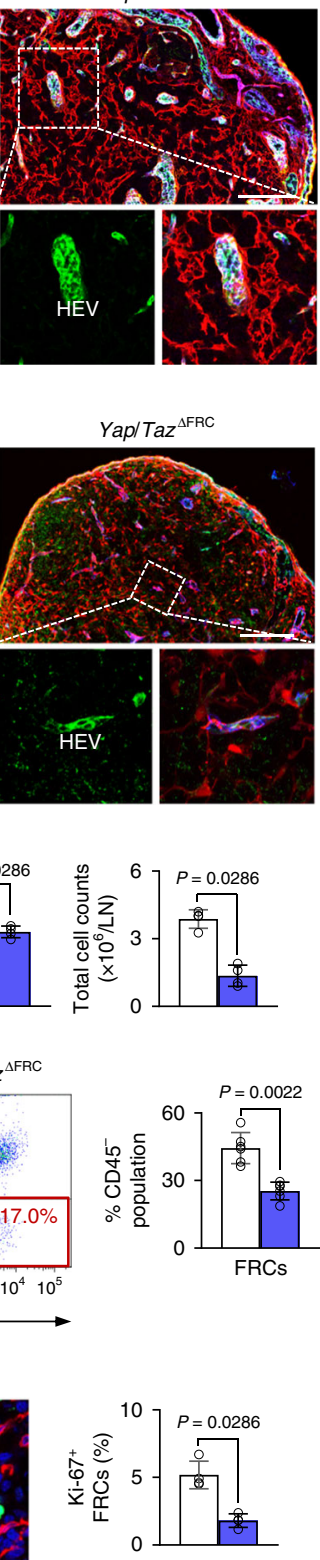
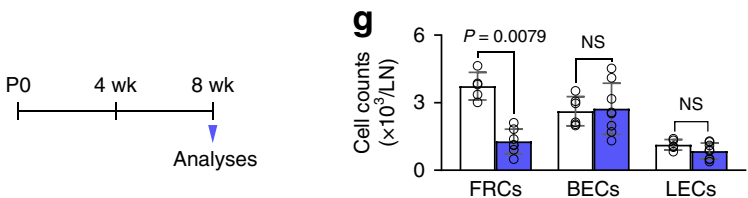

h
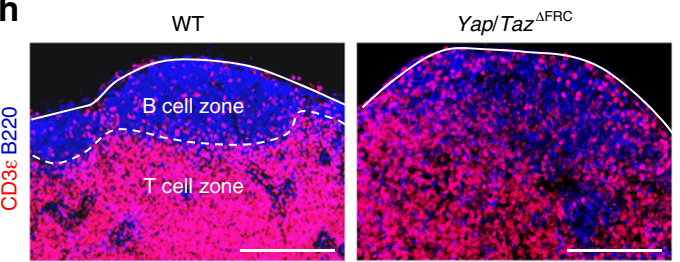

i

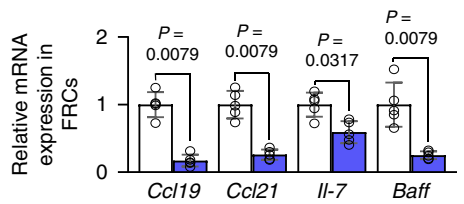

j
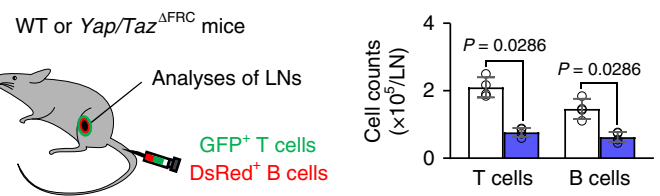

$\mathbf{k}$
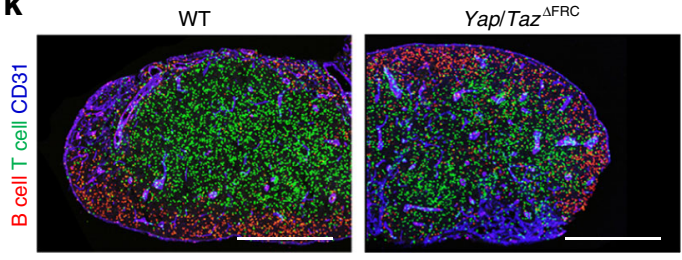

I

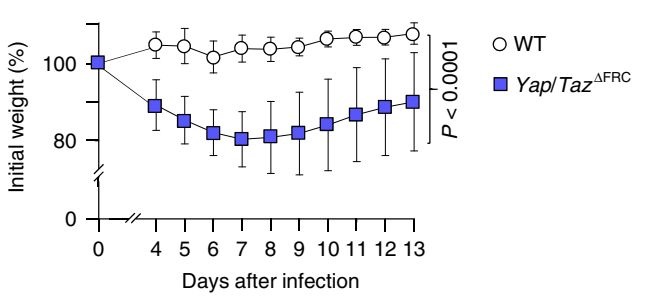

Since immune cells reorganize the reticular network after birth $^{3}$, we examined the recruitment of lymphoid tissue inducer (LTi) cells and the organization of lymphoid tissue organizer (LTo) cells both at the embryonic and postnatal period in Lats $1 / 2^{\Delta \text { FRC }}$ mice (Fig. 2d).

At embryonic (E) day 14.5, engagement of LECs and CD4 ${ }^{+} \mathrm{LTi}$ cells were observed in WT and Lats1/2 $2^{\mathrm{FRC}}$ embryos (Supplementary Fig. 7g), which led to preserved recruitment of LTi cells at m
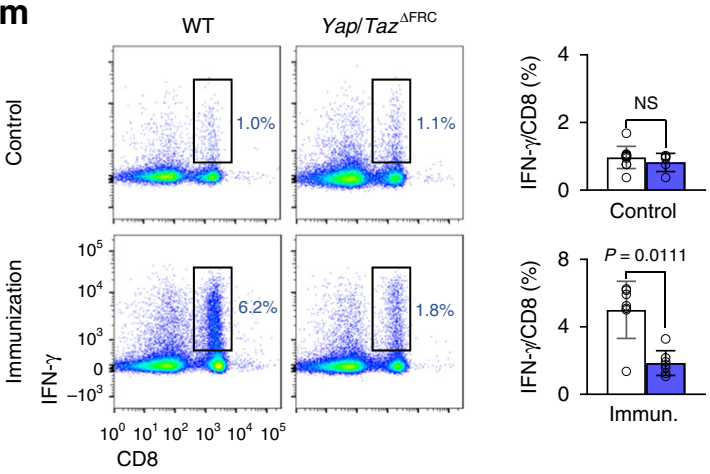

E18.5 (Fig. 2e). Nevertheless, substantial defects in organization of LTo cells were observed at E18.5 and postnatal day (P) 5 in both Yap/Taz ${ }^{\Delta \mathrm{FRC}}$ and Lats $1 / 2^{\Delta \mathrm{FRC}}$ mice compared with WT mice (Supplemental Fig. 7h-j). Thus, adequate activation of YAP/TAZ in FRCs is required for $\mathrm{LN}$ development. Moreover, the distinct border between $\mathrm{B}$ and $\mathrm{T}$ cell zones was disrupted in both Yap/Taz ${ }^{\Delta \mathrm{FRC}}$ and Lats $1 / 2^{\Delta \mathrm{FRC}}$ mice compared with WT mice at P7 (Supplemental Fig. 7k), whereas immune cells were rarely 
Fig. 1 YAP/TAZ support growth and structural organization of LNs by FRCs. a Diagram for generation of indicated mice and their analyses at 8 weeks after birth. b, c Representative images of YAP or TAZ in PDGFR $\beta^{+}$or CCL19+ FRCs in WT and Yap/Taz ${ }^{\triangle F R C}$ mice. FRCs around high endothelial venule (HEV) within the white dashed-line box are magnified in the lower panels with single-channel YAP or TAZ image. Scale bars, $250 \mu$ m. $\mathbf{d}$ Comparisons of body weight, inguinal LN weight and total number of cells within the inguinal LN in WT ( $n=11$; body weight) and Yap/Taz ${ }^{\Delta F C}$ mice ( $n=9$; body weight). e Representative flow cytometric analysis and comparison of proportion of PDPN ${ }^{+}$CD31-FRCs (red box) gated from CD45- stromal cells of skin-draining LNs in WT and Yap/Taz ${ }^{\Delta F R C}$ mice. $\mathbf{f}$ Representative images and comparison of Ki-67+ FRCs (white arrows) in WT and Yap/Taz ${ }^{\Delta F R C}$ mice. Scale bars, $50 \mu \mathrm{m}$. g Comparison of indicated stromal cell counts gated from CD45- cells of skin-draining LNs in WT and Yap/Taz ${ }^{\Delta F R C}$ mice. BECs ( $n=5$ ), blood endothelial cells; LECs $(n=6)$, lymphatic endothelial cells. $\mathbf{h}$ Representative images of distinction between B and T cells (white dashed line) beneath the LN capsule (white line) in WT and Yap/Taz ${ }^{\triangle F R C}$ mice. Scale bars, $200 \mu \mathrm{m}$. i Comparison of indicated mRNA expression in FRCs sorted from WT and $\mathrm{Yap} / \mathrm{Taz}^{\Delta \mathrm{FRC}}$ mice (quintuplicate values using $n=10-15$ mice/group). j, $\mathbf{k}$ Representative images and comparison of DsRed ${ }^{+} \mathrm{B}$ cells and GFP+ T cells within the inguinal $L N$ at $24 \mathrm{~h}$ after the adoptive transfer in WT and $\mathrm{Yap} / \mathrm{Taz}^{\Delta \mathrm{FRC}}$ mice. Scale bars, $500 \mu \mathrm{m}$. I Changes in body weight after $1 \times 10^{3}$ pfu of $\mathrm{A} /$ $\mathrm{PR} / 8$ influenza viral infection $(n=13)$. $\mathbf{m}$ Flow cytometric analyses and comparisons of IFN- $\gamma+\mathrm{CD} 8^{+}$T cells in gated CD3 $\varepsilon^{+} \mathrm{T}$ cells. $n=5$ (CO) or 7 (IM) mice. Unless otherwise denoted, each dot indicates a value obtained from one mouse and $n=4$ mice/group pooled from two independent experiments. Horizontal bars indicate mean \pm SD and $P$ values versus WT by two-tailed Mann-Whitney $U$ test. NS, not significant.

observed in growing LNs of Lats $1 / 2^{\Delta \mathrm{FRC}}$ neonates compared with WT neonates at P14, implying that immune cell recruitment was impaired during the postnatal period in Lats $1 / 2^{\Delta F R C}$ mice (Fig. $2 \mathrm{f}$ ). To evaluate whether the apparent dense distribution of FRCs was due to the reduced number of immune cells, we depleted $\mathrm{T}$ lymphocytes ${ }^{31}$ and other immune cells ${ }^{32}$ by daily injection of antiCD3 $\varepsilon$ mAb into $\mathrm{WT}^{\triangle \mathrm{FRC}-\mathrm{TR}}$ mice (Fig. $2 \mathrm{~g}$ ). Of note, while $\mathrm{T}$ cells were depleted, increase in FRC density was evident in anti-CD3ع $\mathrm{mAb}$-injected mice compared with control mice (Fig. 2h), indicating that impaired immune cell recruitment could conversely affect the density of FRCs during development.

Further analysis of LNs in Lats $1 / 2^{\Delta \mathrm{FRC}}$ mice at P14 showed a reduction in FRCs $(\sim 52 \%)$ but an increase in $\mathrm{PDPN}^{-} / \mathrm{CD}^{-} 1^{-}$ double-negative $(\mathrm{DN})$ populations $(\sim 1.8$-fold) compared with WT mice (Fig. 2i). To investigate whether LN phenotypes of Lats $1 / 2^{\Delta \mathrm{FRC}}$ mice are caused by impaired differentiation of FRCs, we performed lineage-tracing assay using Lats $1 / 2^{\Delta \mathrm{FRC}-\mathrm{TR}}$ mice, generated by crossing Lats $1 / 2^{\Delta \mathrm{FRC}}$ mouse with Rosa26-tdTomato reporter mouse (Fig. 2a). We confirmed enhanced nuclear localization of YAP with upregulated YAP target genes in LN FRCs of Lats $1 / 2^{\triangle \mathrm{FRC}}$ mice and Lats $1 / 2^{\Delta \mathrm{FRC}-\mathrm{TR}}$ mice compared with their controls (Fig. 2j, k). Indeed, we found high expression of Tomato in PDGFR $\beta^{+}$cells within the LNs of Lats1/2 $2^{\Delta \mathrm{FRC}-\mathrm{TR}}$ mice, indicating that these cells arose from FRC precursors (Supplementary Fig. 7l). In addition, Tomato ${ }^{+}$FRCs of Lats1/2 $2^{\Delta \mathrm{FRC}-\mathrm{TR}}$ mice highly expressed aSMA, collagen IV, and PDGFR $\beta$, which are canonical myofibroblastic markers of FRCs but also direct targets of YAP/TAZ ${ }^{33}$ (Fig. 2l). In contrast, expressions of markers of differentiated FRCs including CCL19 and CCL21 were markedly attenuated in FRCs of Lats $1 / 2^{\triangle \mathrm{FRC}-\mathrm{TR}}$ mice compared with WT mice (Fig. 2l, m), suggesting that YAP/TAZ hyperactivation impairs FRC differentiation during development.

Because LATS1/2 can target several pathways ${ }^{25}$, we sought to ascertain if YAP/TAZ are indeed the pivotal target responsible for the aforementioned phenotypes. In this regard, we generated $L 1 / 2-Y / T^{\Delta \mathrm{FRC}}$ mice by crossing Lats $1 / 2^{\Delta \mathrm{FRC}}$ and Yap/Taz ${ }^{\Delta \mathrm{FRC}}$ mice (Fig. 2n, o). Of note, the vessels within the LN and the distinct $\mathrm{B}$ and $\mathrm{T}$ cell zones were partially restored in $L 1 / 2$ $Y / T^{\triangle \mathrm{FRC}}$ mice compared with WT mice (Fig. 2p, q), implying that $\mathrm{YAP} / \mathrm{TAZ}$ are major targets of LATS1/2 in FRC differentiation during LN development.

YAP/TAZ are dispensable in adult mature FRCs. To uncover the roles of YAP/TAZ in adult mature LN FRCs, we generated i-Yap/Taz ${ }^{\triangle \mathrm{FRC}}$ mice by crossing $P d g f r b-C r e E R^{\mathrm{T} 2}$ mouse, for which we confirmed high Cre activity $(\sim 85 \%)$ in PDGFR $\beta^{+}$FRCs (Supplementary Fig. 8a-c), with Yap flox/flox $/$ Taz $^{\text {flox/flox }}$ mouse and administered tamoxifen to 4-weeks old mice and analyzed them after 4 weeks (Fig. 3a). No apparent differences were observed in weight, cellularity, chemokine expression, border of B and T cell zones and distribution of lymphatic vessels in inguinal LNs of Yap/Taz ${ }^{\mathrm{i} \Delta \mathrm{FRC}}$ mice compared with WT mice (Fig. 3b-d and Supplementary Fig. 8d), implying that YAP/TAZ are dispensable for adult mature LN FRCs.

To examine whether the canonical LATS1/2-YAP/TAZ pathway also operates during adulthood, we generated i-L1/2$Y / T^{\Delta \mathrm{FRC}}$ mice by crossing Lats $1^{\text {flox/flox}} /$ Lats $^{\text {flox/flox }}$ mouse with i-Yap/Taz ${ }^{\triangle \mathrm{FRC}}$ mouse, and administered tamoxifen to 6-weeksold mice and analyzed them 2 weeks later (Fig. 3e). Of note, size, weight, cellularity and distribution of PDGFR $\beta^{+}$FRCs in inguinal LNs of $L 1 / 2-Y / T^{\Delta} \triangle \mathrm{FRC}$ mice were comparable to those of WT mice (Fig. 3f, g). Furthermore, although expressions of aSMA, collagen IV, PDGFR $\beta$, and PDPN in LN FRCs were increased in iLats $1 / 2^{\triangle \mathrm{FRC}}$ mice, those in $\mathrm{i}-L 1 / 2-Y / T^{\Delta \mathrm{FRC}}$ mice were comparable to WT mice (Fig. 3h-k). In support of this notion, our ATACsequencing analysis showed enrichment of $P d p n$ promoter region upon Lats1/2 deletion, which, on the other hand, was abrogated upon Yap/Taz deletion (Supplementary Fig. 9a). These results indicate that YAP/TAZ activation must be controlled to maintain the homeostasis of LNs during adulthood.

Identification of YAP/TAZ-regulated pathways in FRCs. To gain insights into roles of YAP/TAZ in FRC differentiation and maturation, we performed transcriptomic analysis of gene expression profiles in isolated FRCs from LNs of WT and Yap/Taz ${ }^{\triangle \mathrm{FRC}}$ mice. Gene Set Enrichment Analysis (GSEA) disclosed significant reductions in genes regulating the epithelialmesenchymal transition (EMT) and E2F target genes together with reductions in YAP target genes in FRCs of Yap/Taz ${ }^{\Delta \mathrm{FRC}}$ compared with WT (Supplementary Fig. 9b,c). Further analysis of FRCs from LNs of i-Lats1/2 $2^{\Delta R C}$ mice showed that genes related to EMT and TGF- $\beta$ signaling were enriched together with upregulation of YAP targets compared with WT mice (Supplementary Fig. 9d,e). Consistently, TGF- $\beta$ signaling-associated genes such as Bmp4, Bmp3, Tgfb2, and $O g n$ and fibrosis-associated genes including Wisp2, Nov, Fgf18, Ctgf, and Pdgfa were ranked among the top 10 upregulators by YAP/TAZ hyperactivation in FRCs (Fig. 31 and Supplementary Fig. 9f). Indeed, genes related with extracellular matrix (ECM), Rho signaling and actin-cytoskeleton rearrangement were upregulated, while those encoding cytokines and chemokines including Ccl19, Ccl21, Il-7, and Baff were downregulated in FRCs of $\mathrm{i}-$ Lats $1 / 2^{\Delta \mathrm{FRC}}$ mice compared with WT mice (Fig. 31, $\mathrm{m}$ and Supplementary Fig. 9g). Ingenuity Pathway Analysis (IPA) revealed that the signaling pathways related to both canonical and non-canonical NF- $\kappa \mathrm{B}$ signaling and production of chemokines were downregulated in LN FRCs of Lats $1 / 2^{\mathrm{i} \Delta \mathrm{FRC}}$ mice compared with WT mice (Fig. $3 \mathrm{~m}$ ). 


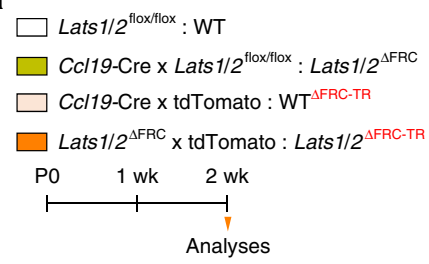

b

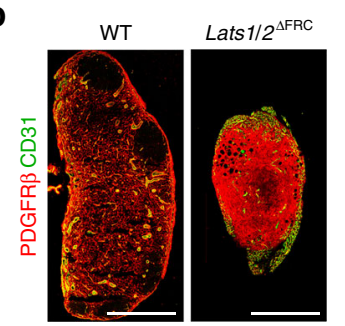

i

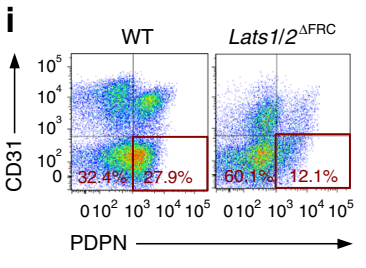

j

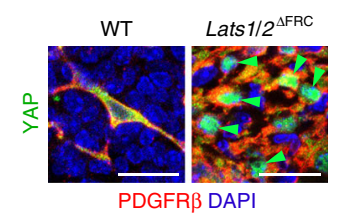

I

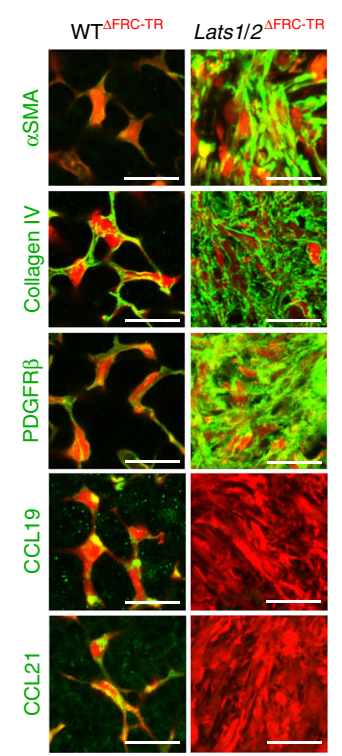

CCL19-Tomato
C
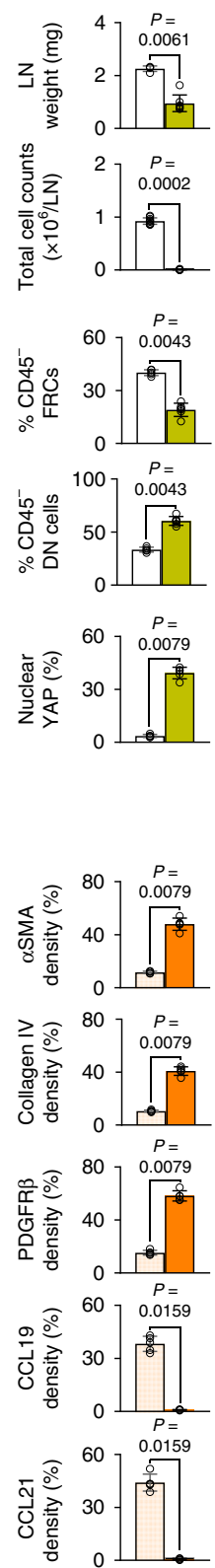

d

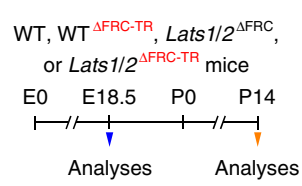

e

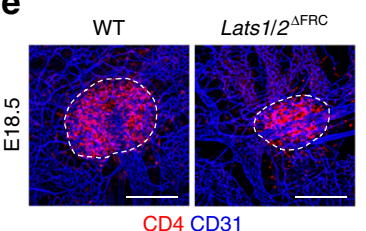

f
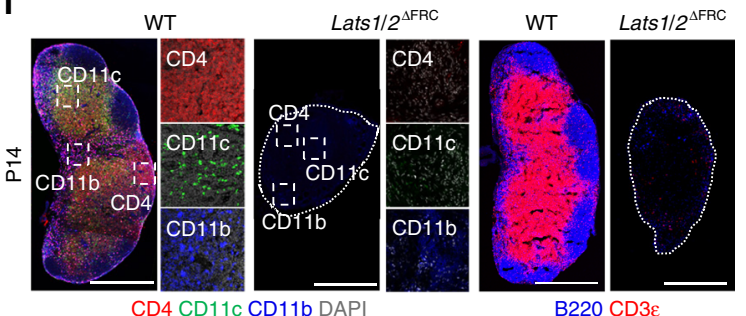

g

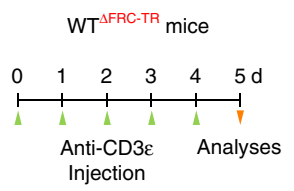

$\mathbf{k}$
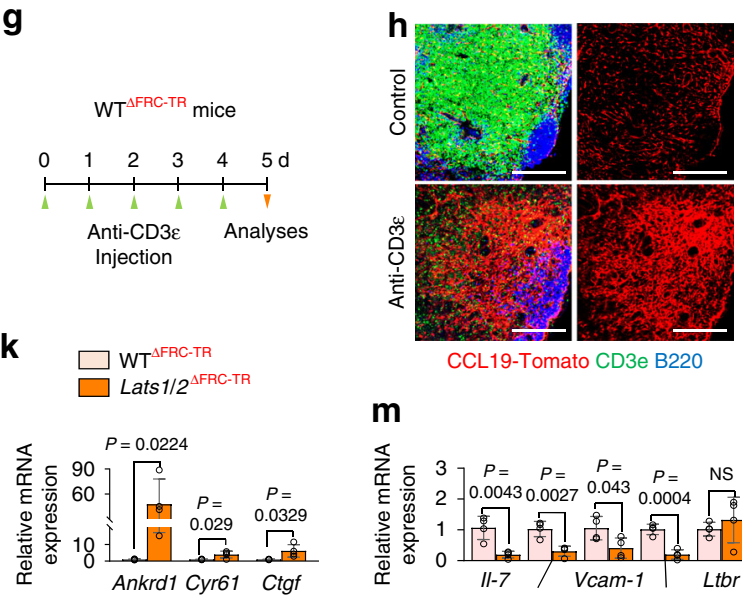

CCL19-Tomato CD3e B220

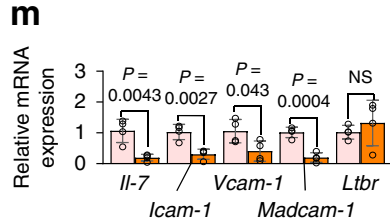

$\mathbf{n}$

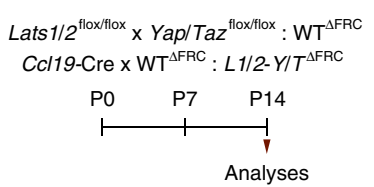

$\mathbf{0}$

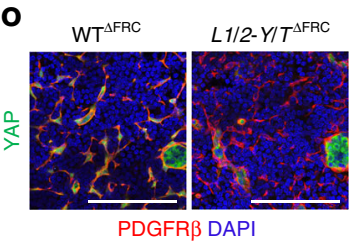

p

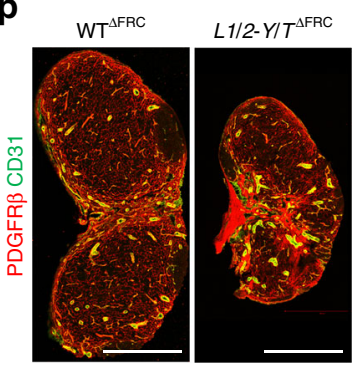

$\mathbf{q}$

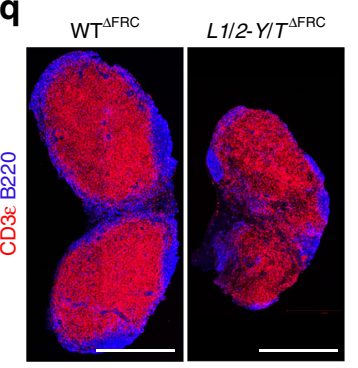

YAP/TAZ hyperactivation in FRCs impedes immune response. To assess whether defects in FRC differentiation influence adaptive immune response in i-Lats $1 / 2^{\Delta \mathrm{FRC}}$ mice, we first examined the expressions of homeostatic chemokines and lymphocyte survival factors in $\mathrm{FRCs}^{6,20}$. Lower mRNA levels of Ccl19, Ccl21, Il-7, and Baff in addition to lower levels of CCL19 and CCL21 were found in LN FRCs of $\mathrm{i}$-Lats $1 / 2^{\Delta \mathrm{FRC}}$ mice compared with WT mice (Supplementary Fig. 10a-d). Although the structures of HEVs were preserved, homing of labeledlymphocytes into the LNs of i-Lats $1 / 2^{\Delta \mathrm{FRC}}$ mice was markedly impaired after the adoptive transfer (Supplementary Fig. 10e-i). When we performed adoptive transfer with CFSE-labeled OT-II $\mathrm{CD}^{+} \mathrm{T}$ cells followed by immunization with ovalbumin (OVA) via footpad injection, both proliferation and activation of OT-II $\mathrm{CD}^{+} \mathrm{T}$ cells were impaired at 3 days after the OVA injection in LNs of $\mathrm{i}$-Lats $1 / 2^{\Delta \mathrm{FRC}}$ mice compared with those of WT mice (Supplementary Fig. 10j-1). Nevertheless, neither alteration in production or differentiation of lymphocytes in bone marrow nor proportion of immune cells including $\mathrm{T}$ and $\mathrm{B}$ lymphocytes in LNs were found (Supplementary Fig. 11a-d). Thus, defects in 
Fig. 2 YAP/TAZ hyperactivation impairs differentiation and maturation of FRCs. a Diagram for analyses of indicated mice at P14. $\mathbf{b}$ Representative images of PDGFR $\beta^{+}$FRCs and CD31+ vessels in WT and Lats1/2 $2^{\triangle F R C}$ mice $(n=5)$. Scale bars, $500 \mu$ m. c Comparisons of LN weight $(n=4-7)$ and total number of cells $(n=6-10)$ in WT and Lats1/2 $\triangle F R C$ mice. d Diagram for analyses of indicated mice at E18.5 or P14. e Representative images of LN anlagen (dashed line) at E18.5 showing CD4+ LTi cells in WT and Lats1/2 ${ }^{\Delta F R C}$ mice $(n=6)$. Scale bars, $200 \mu m$. f, Representative images of indicated markers (dashed box) within the inguinal LN (dotted-line) in WT and Lats1/2 $2^{\Delta F R C}$ mice at P14 $(n=6)$. Scale bars, $500 \mu$ m. $\mathbf{g}$, $\mathbf{h}$ Diagram and representative images for analyses of WT $\triangle$ FRC-TR mice $(n=6)$ that were injected with anti-CD3 $\varepsilon$ for 5 days to induce T cell depletion. Scale bars, $100 \mu \mathrm{m}$. i Representative flow cytometric plots and comparison of proportion of PDPN ${ }^{+}$CD31- FRCs (red box) and PDPN ${ }^{-}$CD31- double-negative (DN) cells of skin-draining LNs in WT and Lats $1 / 2^{\triangle F R C}(n=5-6)$ mice. $\mathbf{j}$ Representative images and comparison of YAP expression and nuclear localization (green-arrowheads) in LN of WT and Lats1/2 $2^{\Delta F R C}$ mice $(n=5)$. Scale bars, $20 \mu \mathrm{m}$. $\mathbf{k}$ Comparison of indicated mRNA expression in FRCs sorted from WT $\Delta$ FRC-TR and Lats $1 / 2^{i \Delta F R C-T R}$ mice $(n=4)$. I Representative images and comparisons of indicated marker expressions in LNs of WT and Lats $1 / 2^{\Delta F R C}$ mice $(n=4-5)$. Scale bars, $20 \mu \mathrm{m}$.

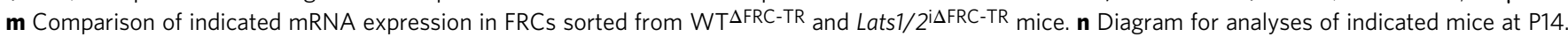
- Representative images of YAP expression in LNs of WT and $L 1 / 2-Y / T^{\Delta F R C}$ mice. Scale bars, $100 \mu \mathrm{m}$. $\mathbf{p}$, $\mathbf{q}$ Representative images of indicated markers in $L N$ s of $W T$ and $L 1 / 2-Y / T \Delta F R C$ mice. Scale bars, $500 \mu \mathrm{m}$. Unless otherwise denoted, each dot indicates a value obtained from inguinal $L N$ and $n=4$ mice. Horizontal bars indicate mean \pm SD and $P$ values versus WT or WT $\triangle$ FRC-TR by two-tailed Mann-Whitney $U$ test except for (k) and (m) (two-tailed Student's $t$-test). NS, not significant.

FRC differentiation by YAP/TAZ hyperactivation primarily results in impaired adaptive immune response.

YAP/TAZ are activated in myofibroblastic FRC precursors. Of note, LT $\beta R$ signaling, which is known to regulate FRC maturation ${ }^{20,21}$, was predicted to be preferentially and significantly influenced by YAP/TAZ hyperactivation in FRCs by our IPA analysis (Fig. $3 \mathrm{~m}$ ). We therefore sought to corroborate the interaction between LT $\beta R$ and YAP/TAZ in FRCs by generating $L T b R^{\triangle F R C-Y R}$ mice by crossing Ccl19-Cre mouse with $L t b r^{\text {flox/flox }}$ and YFP reporter mouse (Fig. 4a). Consistent with previous reports ${ }^{20,21}$, levels of $\alpha$ SMA, PDGFR $\beta$ and collagen IV in FRCs were increased, while levels of LTRR, CCL19 and CCL21 were decreased in $L t b r \Delta \mathrm{FRC}$ mice compared with those of WT mice (Fig. 4b). Importantly, YAP/TAZ were nuclear localized and expressions of YAP target genes were upregulated in FRCs of $L t b r \Delta \mathrm{FRC}$ mice compared with WT mice (Fig. 4c, d), suggesting that YAP/TAZ activity could be negatively regulated by LT $\beta R$ signaling.

To evaluate whether YAP/TAZ hyperactivation-induced myofibroblastic phenotypes in vivo are mediated through FRCintrinsic signaling, we cultured primary FRCs derived from iLats $1 / 2^{\triangle \mathrm{FRC}-\mathrm{TR}}$ mice. Following 4-hydroxytamoxifen (4-OHT) treatment, efficient ( $\sim 83.7 \%)$ depletion of LATS1/2 and upregulation of YAP target genes were confirmed compared with control EtOH treatment (Fig. 4e-g and Supplementary Fig. 12a,b). In this condition, levels of pro-fibrotic markers such as vimentin and collagen $\mathrm{I}^{34}$ were increased, and contractility was potentiated as shown as enhanced assembly of F-actin (Fig. 4h, i and Supplementary Fig. 12c). To see whether this finding can be recapitulated in human, we cultured primary FRCs derived from human normal LNs. Primary cultured human FRCs transfected with adenovirus encoding constitutive active form of YAP (YAP5SA) or TAZ (TAZ4SA) enhanced the levels of aSMA, vimentin and collagen I compared with those transfected with control vector (Fig. $4 \mathrm{j}-\mathrm{l}$ ), indicating that phenotypes of YAP/TAZ hyperactivation in FRCs are instrinsic.

YAP/TAZ regulate chemokine expression before LT $\beta R$ engagement. To examine the regulatory mechanism between LT $\beta R$ and YAP/TAZ, primary cultured FRCs derived from WT mice were stimulated with an agonistic LT $\beta$ R antibody (Supplementary Fig. 13a,b). As expected, the stimulation of LT $\beta R$ increased NIK protein but reduced p100 protein in time and dose-dependent manners (Supplementary Fig. 13b,c). Of note, it not only increased p52 protein level, but also promoted activity of LATS and increased pYAP/YAP ratio (Fig. 5a). Importantly, nuclear-cytoplasmic fractionation and immunofluorescence analyses revealed that the LT $\beta R$ stimulation promoted nuclear to cytoplasmic shuttling of YAP/TAZ
(Fig. 5b and Supplementary Fig. 13d). Thus, LT $\beta$ R signaling suppresses YAP/TAZ activity in FRCs.

LT $\beta R$ intracellular signaling mainly takes a non-canonical NF$\kappa B$ pathway through $p 52 /$ RelB to exert its cellular functions ${ }^{35,36}$. To examine whether YAP/TAZ physically interact with either p52 or RelB, we performed immunoprecipitation analysis using antiYAP/TAZ antibody in primary cultured FRCs derived from WT mice. p52 was readily detected in the immuno-complexes pulled with anti-YAP/TAZ antibody but not in those from control IgG (Fig. 5c). To ensure this finding, we transfected HEK293T cells with a gene encoding streptavidin-binding peptide-tagged constitutively active form of TAZ (SBP-TAZ4SA) and pulled all bound proteins using the streptavidin resin. We confirmed presence of both $\mathrm{p} 52$ and RelB in the resin, and stabilization of p52 protein through TAZ (Fig. 5d and Supplementary Fig. 13e).

YAP/TAZ contain WW domains that bind to PPxY motif of the binding protein partners ${ }^{25,37}$. Although neither p52 nor RelB has PPxY motif, both p52 and RelB have highly conserved PPY motifs 38,39 , which could be possible binding targets for YAP/ TAZ. Of note, point mutation of the PPY motif of p52 to PPA (p52-Y293A) completely abrogated the binding interaction with SBP-TAZ4SA (Fig. 5e), whereas the RelB mutants (RelB-Y248A and RelB-Y341A) still showed interaction with SBP-TAZ4SA (Supplementary Fig. 13f). Conversely, deleting the WW domain in SBP-TAZ4SA (TAZ4SA $\triangle W W$ ) markedly attenuated its binding with $\mathrm{p} 52$, but again this did not affect the binding with RelB (Fig. 5f), indicating that TAZ independently binds to both RelB and p52. To elucidate how this molecular interaction regulates FRC differentiation, we transfected HEK293T cells retaining a Ccl19 promoter-driven luciferase with the gene encoding either TAZ4SA, TAZ4SA $\triangle W W$, p52, or p52-Y293A (Fig. 5g). Intriguingly, while a single transfection of p52 enhanced the luciferase activity by 7.5 -fold, co-transfection with TAZ4SA and $\mathrm{p} 52$ promoted the luciferase activity by 13.5 -fold (Fig. $5 \mathrm{~h}$ ). However, this increase in luciferase activity was not observed in cells that were transfected with either TAZ4SA $\triangle W W$, p52Y293A or both of these (Fig. 5h). We further validated our findings in primary cultured FRCs derived from i-Yap/Taz ${ }^{\triangle \mathrm{FRC}}$ mice, where p52-regulated transcripts such as Ccl19 and Ccl21 were markedly attenuated by $4-\mathrm{OHT}$ treatment compared with EtOH (Supplementary Fig. 13g,h). Conversely, in situ proximity ligation assay and chromatin immunoprecipitation (ChIP) analysis revealed that LT $\beta$ R stimulation promoted nuclear to cytoplasmic shuttling of YAP/TAZ-p52 complex and attenuated its binding affinity to promoter regions of $C c l 19$ (Fig. 5i, j). Thus, proper interaction between YAP/TAZ and p52 seems to be required for the expression of chemokines such as $\mathrm{Ccl} 19$ before LT $\beta R$ engagement (Supplementary Fig. 13i). 
a

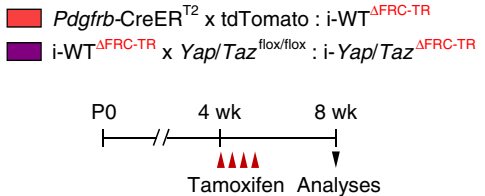

b
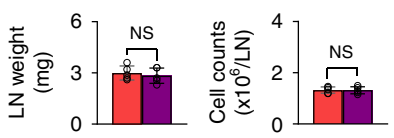

d

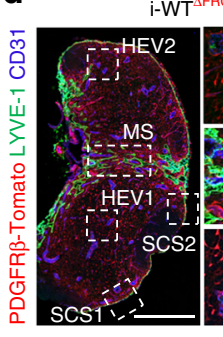

$-W T^{\triangle F R C-T R}$

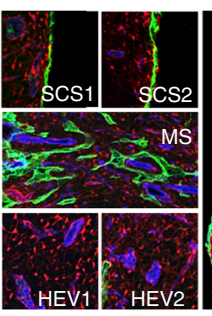

h

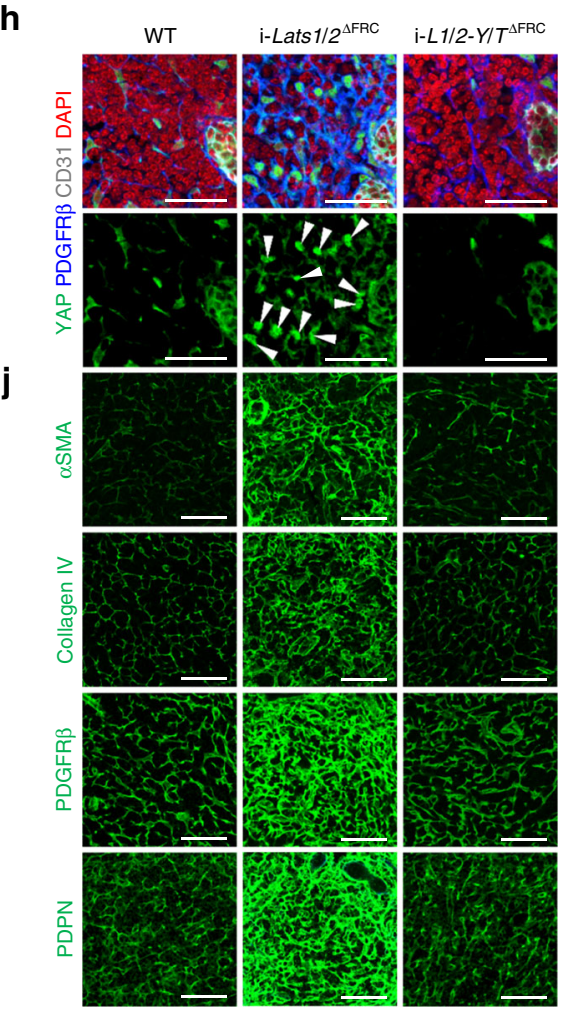

e

$$
\begin{aligned}
& \square \text { Lats } 1 / \text { Lats } 2^{\text {floxflox }}: \text { WT }
\end{aligned}
$$

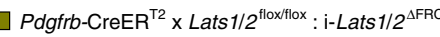

$$
\begin{aligned}
& \text { Lats } 1 / 2^{\mathrm{i} A \mathrm{FAC}} \times \text { Yap/Taz }{ }^{\text {floxflox }}: \mathrm{i}-L 1 / 2-Y / T^{\Delta \mathrm{FRC}}
\end{aligned}
$$

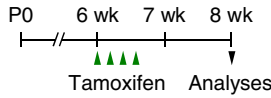

f
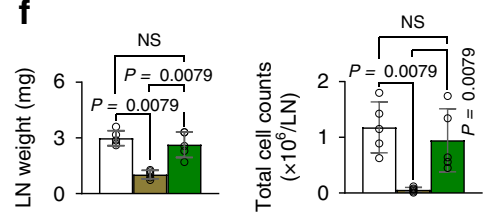

g

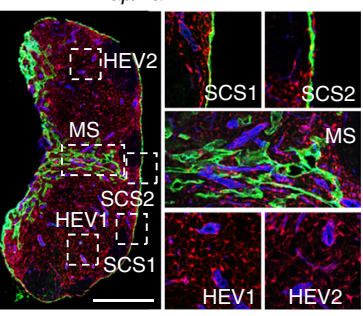

i

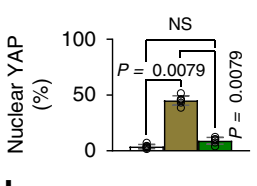

k

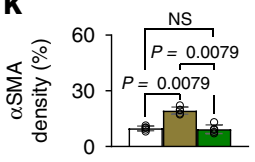

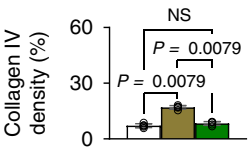
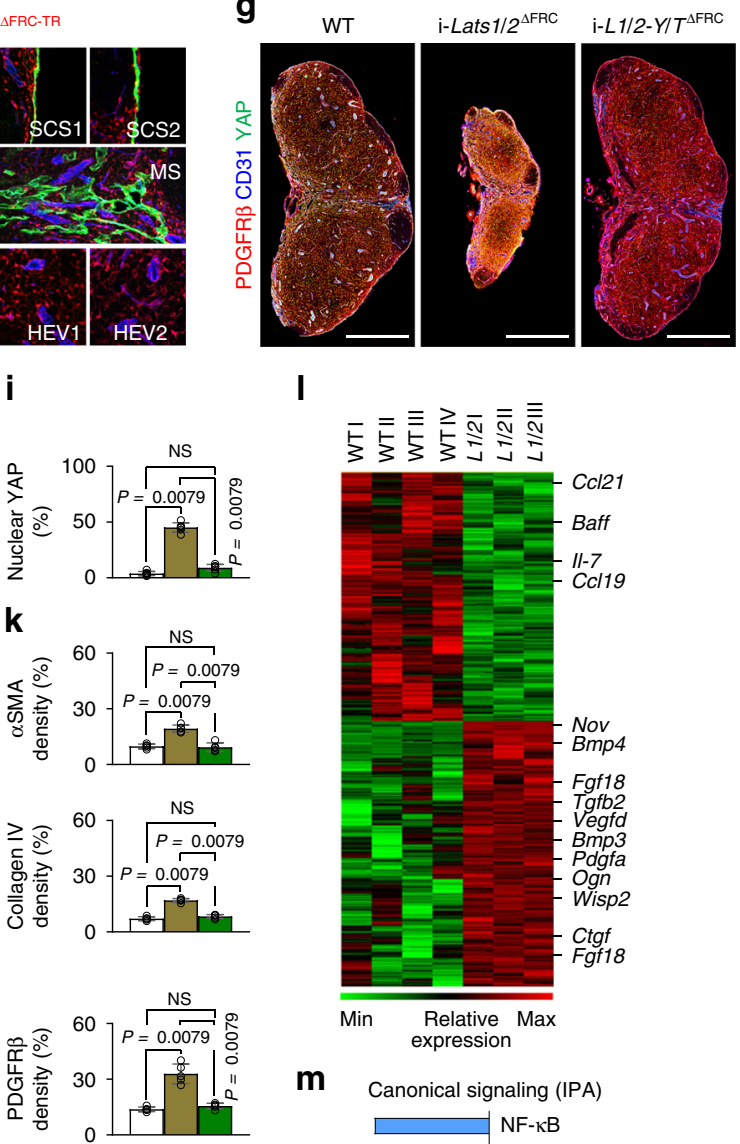

I

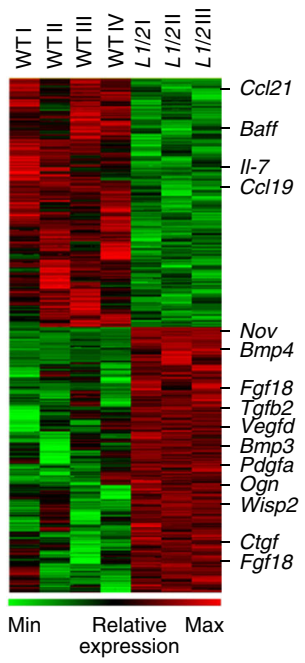

m Canonical signaling (IPA)

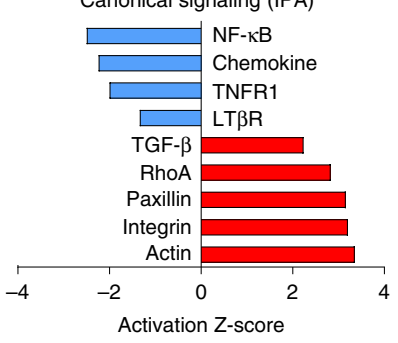

YAP/TAZ drive specification of mesenchymal cells into FRCs. These observations led us to postulate that the binding of YAP/ TAZ to p52 is required in myofibroblastic FRC precursors before LT $\beta R$ engagement. To verify this postulation, we depleted $\mathrm{Yap} / \mathrm{Taz}$ in myofibroblastic FRC precursors ( $L t b r^{\Delta \mathrm{FRC}-\mathrm{YR}}$ ) by generating $L t b r-Y / T^{\Delta \mathrm{FRC}-\mathrm{YR}}$ mice, and we also generated i- Ltbr$Y / T^{\Delta \mathrm{FRC}-\mathrm{YR}}$ mice by crossing $P d g f r b$-CreER ${ }^{\mathrm{T} 2}$-YFP reporter mouse (i-WT $\Delta$ FRC-YR) with $L t b r^{\text {flox/flox }}\left(\mathrm{i}-L t b r^{\Delta \mathrm{FRC}-\mathrm{YR}}\right.$ ) or $Y a p$ flox/flox $/ T a z^{\text {flox/flox }}$ mouse (i-Y/T $\Delta$ FRC-YR) (Fig. 6a). Surprisingly, the skin-draining LNs including inguinal, axillary and brachial

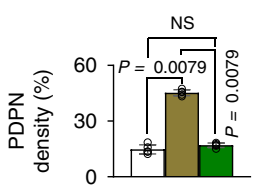

LNs of $L t b r-Y / T^{\Delta \mathrm{FRC}-\mathrm{YR}}$ and $\mathrm{i}-L T b R-Y / T^{\Delta \mathrm{FRC}-\mathrm{YR}}$ mice had perilipin $^{+}$adipocytes, which constitutes $\sim 20-25 \%$ of the LN density (Fig. 6b, c and Supplementary Fig. 14a).

These aberrant adipocytes were mainly located along the infiltrating lymphatic vessels within the skin-draining LNs, while no adipocytes were observed within the mesenteric LNs of Ltbr$Y / T^{\Delta \mathrm{FRC}-\mathrm{YR}}$ and i-Ltbr-Y/T $\Delta \mathrm{FRC}-\mathrm{YR}$ mice (Supplementary Fig. 14a). Of note, all the perilipin ${ }^{+}$or BODIPY $^{+}$adipocytes were $\mathrm{YFP}^{+}$in LNs of $L t b r-Y / T^{\Delta \mathrm{FRC}-\mathrm{YR}}$ and $\mathrm{i}-\mathrm{L} t b r-Y / T^{\Delta \mathrm{FRC}-\mathrm{YR}}$ mice, indicating that they originated from $\mathrm{YFP}^{+}$FRC precursors 
Fig. 3 Canonical Hippo pathway LATS1/2-YAP/TAZ governs FRCs. a Diagram for generation of indicated mice and their analyses at 8-weeks old after the tamoxifen injection from 4-weeks old. b Comparisons of the inguinal LN weight and cellularity within the inguinal LN in i-WT $\triangle F R C-T R$ and i-Yap/Taz ${ }^{\Delta F R C-T R}$ mice. c Representative images of intact border between B and T cell zones (white dashed line) beneath the LN capsule (white line) in i-WT $\triangle F R C-T R$ and

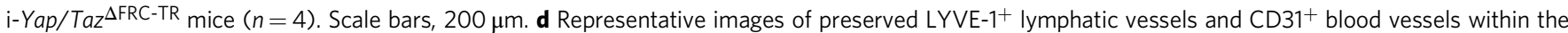
inguinal LN in i-WT ${ }^{\triangle F R C-T R}$ and i-Yap/Taz ${ }^{\Delta R R C-T R}$ mice $(n=4)$. The regions within the white dashed-line box around subcapsular sinuses $(S C S)$, medullary sinus (MS) and HEVs are magnified as indicated. Scale bars, $500 \mu \mathrm{m}$. e Diagram for generation of indicated mice for their analyses at 8-weeks old after the tamoxifen delivery from 6-weeks old. f Comparisons of the inguinal LN weight and total number of cells within the inguinal LN in WT, i-Lats $1 / 2^{\Delta \mathrm{FRC}}$ or i-L1/2-Y/T $\triangle F R C$ mice. $\mathbf{g}$ Representative images of inguinal LN in WT, i-Lats $1 / 2^{\Delta F R C}$ or i-L1/2-Y/T $\triangle F R C$ mice. Scale bars, $500 \mu \mathrm{m}$. $\mathbf{h}, \mathbf{i}$ Representative images and comparison of YAP nuclear localization (white arrowheads) in inguinal LN of WT, i-Lats $1 / 2^{\Delta F R C}$ or i-L1/2-Y/T $\Delta F R C$ mice. Scale bars, $40 \mu m$.

j, $\mathbf{k}$ Representative images and comparisons of indicated marker expressions in FRCs around T cell zone of inguinal LN in WT, i-Lats $1 / 2 \triangle F R C$ or $i-L 1 / 2$ $Y / T^{\triangle F R C}$ mice. Scale bars, $60 \mu \mathrm{m}$. I Heatmap and hierarchical clustering of differentially expressed genes of RNA-Seq data in isolated FRCs from WT and $\mathrm{i}$-Lats $1 / 2^{\triangle \mathrm{FRC}}$ mice and list of selected downregulated genes (green) encoding cytokines and chemokines and upregulated genes (red) involved in TGF- $\beta$ signaling. $\mathbf{m}$ Canonical IPA-annotated pathways listed in absolute IPA activation Z-score $(P<0.05)$ to identify potential activation or inhibition of indicated signaling pathways in isolated FRCs from i-Lats1/2 $\triangle F R C$ mice compared with WT. Unless otherwise denoted, each dot indicates a value obtained from one mouse and $n=5$ mice/group pooled from two independent experiments. Horizontal bars indicate mean $\pm \mathrm{SD}$ and $P$ values versus WT, i-WT $\triangle F R C-T R$ or i-Lats $1 / 2^{\triangle F R C}$ by two-tailed Mann-Whitney $U$ test. NS, not significant.

(Fig. 6d, e). Further analysis with primary cultured FRCs derived from $L t b r-Y / T^{\Delta \mathrm{FRC}-\mathrm{YR}}$ mice showed upregulation of adipogenic genes such as Pparg and Cebpa ${ }^{40}$ without ectopic fat accumulation (Fig. 6f, g, and Supplementary Fig. 14b), suggesting enhanced adipogenic activity of FRCs rather than metabolic dysregulation in $L t b r-Y / T^{\Delta \mathrm{FRC}-\mathrm{YR}}$ mice.

It has been proposed that both adipocytes and LN stromal cells are mesenchymal origin and developmentally related ${ }^{12}$. We therefore examined whether Hippo signaling determines the fate specification of mesenchymal stem cells by utilizing C3H10T1/2 cells (Fig. 6h). Adipogenic culture of C3H10T1/2 cells led to their differentiation into adipocyte-lineage cells, but TAZ4SA overexpressing $\mathrm{C} 3 \mathrm{H} 10 \mathrm{~T} 1 / 2$ cells significantly abrogated adipogenesis while they expressed enhanced FRC commitment markers ${ }^{19,41}$ (Fig. 6i-k). These data indicate that YAP/TAZ drive specification and differentiation of mesenchymal cells into FRCs, while they suppress those into other cell types including adipocytes.

\section{Discussion}

FRCs discard some of their myofibroblastic characters as they differentiate and complete maturation, while they gain chemokine secretory functions which are essential for organizing adaptive immunity ${ }^{12,20}$. Here, we show that the canonical Hippo pathway, together with LT $\beta \mathrm{R}$-non-canonical NF- $\kappa \mathrm{B}$ signaling, critically regulates differentiation and maintenance of LN FRCs (Fig. 6l). Deletion of YAP/TAZ in FRCs during development impairs their growth and differentiation, compromising the structural organization of LNs. Hyperactivation of YAP/TAZ in FRCs during the developmental period severely impairs differentiation and maturation of FRCs, leaving non-functional and fibrotic LNs constituted with immature FRCs. Even when YAP/TAZ are hyperactivated in adult mature FRCs, enhanced myofibroblastic characters of FRCs and severe LN fibrosis are similarly observed. These alterations ultimately lead to substantial distortion of LN microarchitecture with impairs adaptive immune responses. Although depletion or hyperactivation of YAP/TAZ leads to similar phenotypes, outcomes of YAP/TAZ depletion are due to loss of FRC pool, while those of YAP/TAZ hyperactivation are owed to maturation defects of FRCs.

These results show that proper modulation of FRCs by canonical Hippo signaling is critical for formation and maintenance of LNs during development and in adult. A previous study proposes that mesenchymal stem cells are differentiated into mature FRCs by suppressing their adipogenicity through promotion of LT $\beta \mathrm{R}$ p52/RelB signaling ${ }^{12}$. TAZ binding to PPAR $\gamma$ via its WW domain is also demonstrated to inhibit adipogenesis ${ }^{42}$. However, the association between LT $\beta R$ signaling and YAP/TAZ has not been previously addressed. Our biochemical analyses demonstrate that YAP/TAZ and p52 form a complex to regulate the expression of chemokines such as CCL19 in FRC precursors. Then, upon LT $\beta R$ activation, YAP/TAZ become phosphorylated and translocated to the cytoplasm, leading to the maturation of FRC precursors. While previous studies demonstrate that LT $\beta \mathrm{R}$ signaling is critical for FRC differentiation, neither ablation of LT $\beta R$ nor its ligands in FRC precursors is critical for maintaining the FRC lineage ${ }^{19-21}$. Here, we show that depletion of YAP/TAZ in LT $\beta R$ ablated FRC precursors induces the transition of the FRC lineage into adipocytes. Collectively, these results indicate that LT $\beta R$ signaling and YAP/TAZ play both overlapping and independent roles for cell commitment and maintenance of FRCs.

Still, questions remain in linking Yap/Taz modulation in FRCs with its final outcomes. In case of Yap/Taz depletion during development, it results in decreased cellularity of FRCs, which lead to shortage in conduit coverage. Considering that this study agrees with a previous study in that FRC coverage of the LN conduit is not critical for the maintenance of conduit integrity and function ${ }^{17}$, the purpose of FRC coverage around conduits needs further investigation. In contrast, Yap/Taz activation during development lead to maturation defects, and as a consequence impairs LN microarchitecture, which itself could not fully explain the lethal phenotypes. Therefore, the effects of Yap/Taz modulation in other FRC subsets ${ }^{43,44}$ in lymphoid tissues such as spleen and thymus remains to be studied. In addition, exploiting a more specific inducible Cre driver would provide better understanding when comparing the consequences of constitutional or conditional YAP/TAZ manipulation in FRCs. In conclusion, our study clearly demonstrates that the Hippo pathway plays pivotal roles in maturation and maintenance of FRCs of LNs.

\section{Methods}

Mice. Specific pathogen-free (SPF) C57BL/6J mice (\#000664), Rosa26-tdTomato mice (\#007914), Rosa26-eYFP (\#007903), Actb-DsRed (\#006051), Actb-GFP (\#003291), Lgr5-Cre (\#008875), and OT-II (\#004194) mice were purchased from the Jackson Laboratory. Lats $1^{\text {flox/flox } 29}$, Lats $2^{\text {flox/flox 30, }}$ Yap flox/flox 27, Taz $^{\text {flox/flox } 28}$ $L t b r$ flox/flox 20, Ccl19-Cre ${ }^{20}$, and Pdgfrb-Cre-ER ${ }^{\mathrm{T} 2} 45$ mice were transferred, established, and bred in SPF animal facilities at KAIST. All mice were maintained in the C57BL/ 6 background and fed with free access to a standard diet (PMI LabDiet) and water. In order to induce Cre activity in the Cre-ER ${ }^{\mathrm{T} 2}$ mice, $2 \mathrm{mg}$ of tamoxifen (Sigma-Aldrich) was dissolved in corn oil (Sigma-Aldrich) and intra-peritoneally (i.p.) injected at indicated time points. All mice were anesthetized with i.p. injection of a combination of anesthetics $(80 \mathrm{mg} / \mathrm{kg}$ ketamine and $12 \mathrm{mg} / \mathrm{kg}$ of xylazine) before being euthanatized. We complied with all ethical regulations for animal testing and research and performed all animal experiments and euthanasia under the approval from the Institute Animal Care and Use Committee (No. KA2016-12) of Korea Advanced Institute of Science and Technology (KAIST). Mouse model nomenclatures are included in Supplementary Table 1. 
a

$L$ tbr ${ }^{\text {floxfliox }}: \mathrm{WT}^{\Delta \mathrm{FRC}}$

CCl19-Cre $x$ Ltbriloxflox $:$ Ltbr ${ }^{A R R C}$

CCl19-Cre $\times$ YFP : WT ${ }^{\triangle F R C-Y R}$

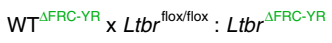

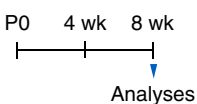

b
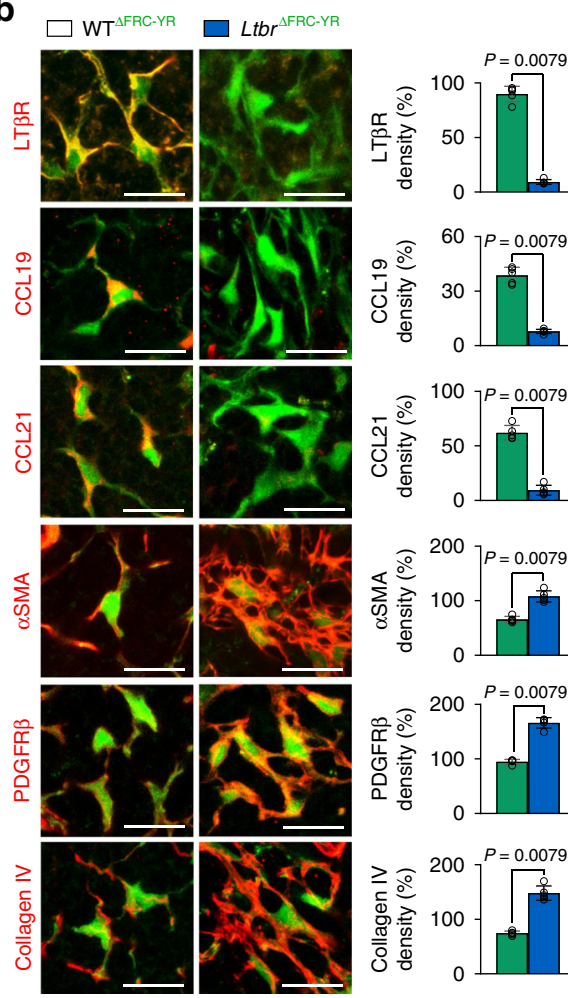

CCL19-YFP

C
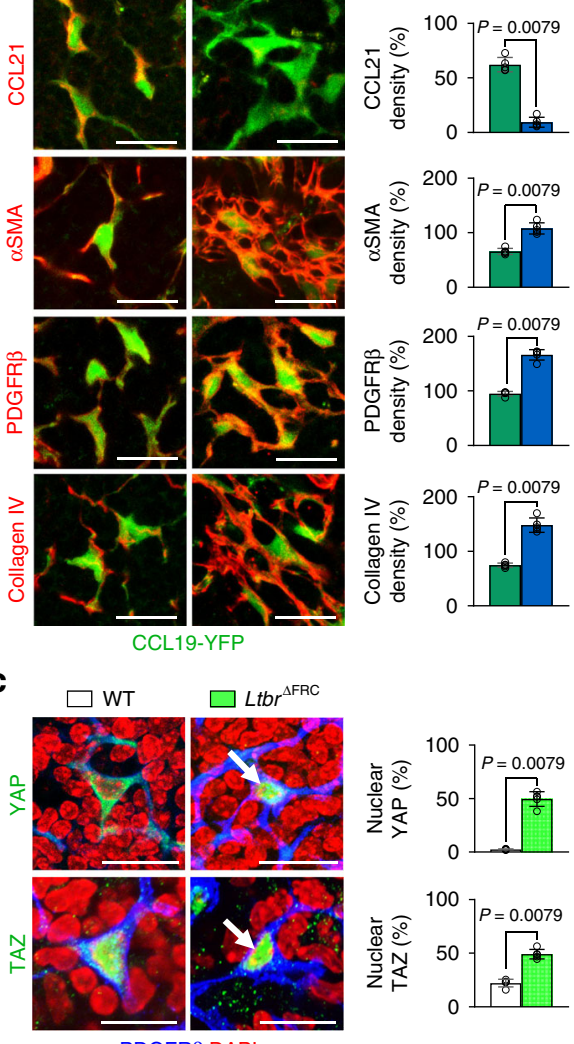

e

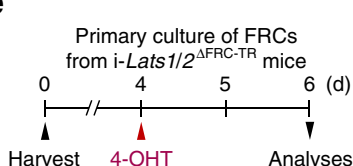

f EtOH 4-OHT

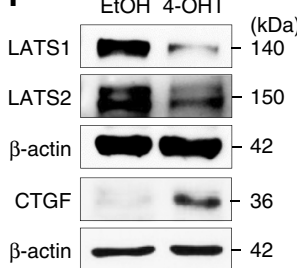

g

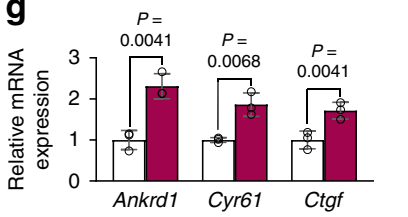

$\square \mathrm{EtOH}$

$\square$ 4-OHT

h
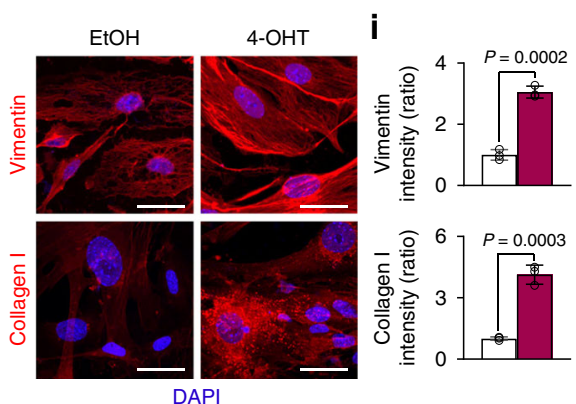

j

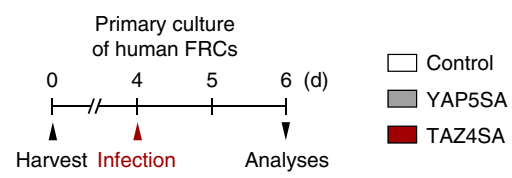

$\mathbf{k}$
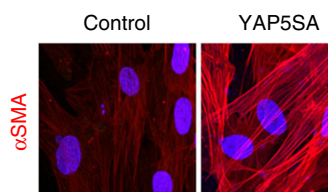

TAZ4SA
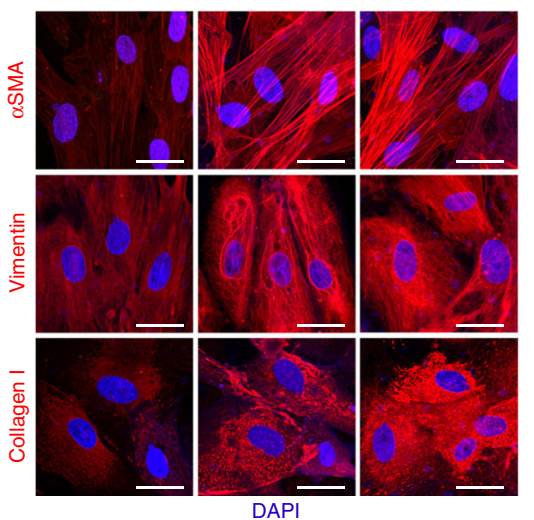

DAPI

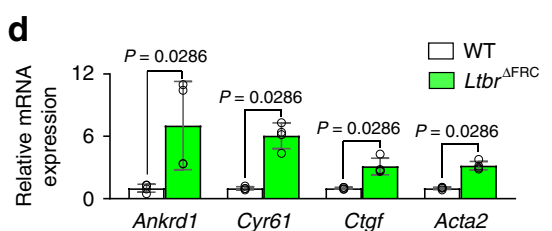

Histological analyses. Gross images of LNs were acquired using AxioZoom V16 stereo zoom microscope (Carl Zeiss). For LN weight measurement, bilateral inguinal LNs were pooled and weighed using an analytical balance (Mettler Toledo). For immunofluorescence staining of LNs, harvested samples were fixed in $1 \%$ paraformaldehyde (PFA) in PBS overnight at $4{ }^{\circ} \mathrm{C}$ and dehydrated in $20 \%$ sucrose in PBS overnight at $4{ }^{\circ} \mathrm{C}$. Samples were embedded in tissue freezing medium (Leica) and frozen blocks were cut into $20-\mu \mathrm{m}$-thick sections. Samples were blocked with $5 \%$ goat (or donkey) serum in $0.3 \%$ Triton X-100 in PBS (PBST) and incubated for overnight at $4{ }^{\circ} \mathrm{C}$ with primary antibodies (diluted at a ratio of 1:200 in blocking solution). After several washes with PBS, samples were incubated for $2 \mathrm{~h}$ at RT with secondary antibodies (diluted at a ratio of 1:1000 in blocking solution). After washing several times with PBS, samples were mounted with fluorescent mounting medium (DAKO) and images were acquired using LSM780 or LSM880 confocal microscope (Carl Zeiss).

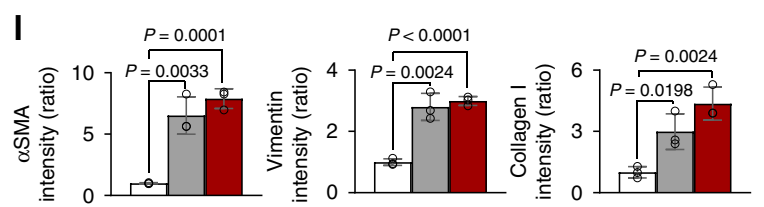

To examine YAP and TAZ distribution in human LNs, several cervical LNs around thyroid papillary carcinoma were collected from the patients undergoing thyroidectomy with written informed consent according to the protocol approved by the institutional review board of Pusan National University (H-1610-002-003) and Samsung Medical Center (2018-06-061).

For whole-mount staining of embryo, pregnant mice were anesthetized at the indicated day of embryonic development after vaginal plug, and the uteri were removed. Embryos were dissected and the yolk sacs were used for verification of genotype. Inguinal fat pads and LN anlagen were isolated together with the

covering skin and processed for staining. Samples were fixed in 2\% PFA for $2 \mathrm{~h}$ at $4{ }^{\circ} \mathrm{C}$ and washed several times with PBS for the staining.

For staining of primary cultured FRCs, cells were plated in 8-well Nunc LabTek II chamber slides (Sigma-Aldrich) and fixed with $4 \%$ PFA for $8 \mathrm{~min}$ at RT. After several washes with PBS, samples were blocked with $5 \%$ goat (or donkey) 
Fig. 4 FRC-specific depletion of Ltbr activates YAP/TAZ-induced myofibrosis. a Diagram for generation of indicated mice and their analyses at 8-weeks old. b Representative images and comparisons of indicated marker expressions on CCL19-YFP + FRCs in WT $\triangle F R C-Y R$ and Ltbr $\triangle F R C$-YR mice. Scale bars, $20 \mu \mathrm{m}$. c Representative images and comparisons of YAP and TAZ nuclear localization (white arrows) in inguinal LN of WT $\triangle F R C$ and $L t b r \Delta F R C$ mice. Scale bars, $20 \mu \mathrm{m}$. d Comparison of indicated mRNA expression in FRCs sorted from WT $\triangle \mathrm{FRC}$ and $L$ tbr $\triangle \mathrm{FRC}$ mice. Each dot indicates a mean of quadruplicate values using $n=8-12$ mice/group from three independent experiments. e Diagram for primary culture of FRCs derived from i-Lats $1 / 2^{\Delta F R C-T R}$ mice and

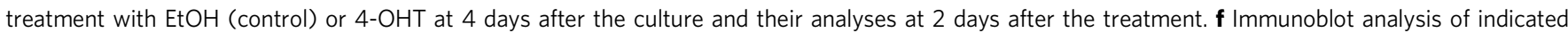
proteins in primary cultured mouse FRCs after treatment with EtOH or 4-OHT for 2 days. $\mathbf{g}$ Comparisons of indicated mRNA expression normalized to Gapdh in primary cultured mouse FRCs after treatment with $\mathrm{EtOH}$ or 4-OHT for 2 days. Each dot indicates a mean of triplicate values from three independent experiments. $\mathbf{h}$, i Representative images and comparisons of indicated marker expressions in primary cultured mouse FRCs after treatment with EtOH or 4OHT for 2 days. Scale bars, $30 \mu \mathrm{m}$. Each dot indicates a mean of triplicate values from three independent experiments. $\mathbf{j}$ Diagram for primary culture of human FRCs for 4 days and infection with an adenovirus to induce overexpression of active YAP (YAP5SA) or TAZ (TAZ4SA) for their analyses at 2 days after the infection. $\mathbf{k}$, I Representative images and comparisons of indicated marker expressions in primary cultured human FRCs infected with control-, YAP5SA-, or TAZ4SA-adenovirus. Scale bars, $30 \mu \mathrm{m}$. Each dot indicates a mean of triplicate values from three independent experiments. Unless otherwise denoted, each dot indicates a value obtained from one mouse and $n=5$ mice/group pooled from two independent experiments. Horizontal bars indicate mean \pm SD and $P$ values versus WT $\triangle F R C$ or WT $\triangle F R C$-YR by two-tailed Mann-Whitney $U$ test except for (g), (i), and (I) (two-tailed Student's t-test). NS, not significant.

serum in PBST for 30 min at RT. Cells were incubated with primary antibodies (diluted at a ratio of 1:200 in blocking solution) for overnight at $4{ }^{\circ} \mathrm{C}$. The following primary and secondary antibodies were used in the immunostaining: anti-YAP (rabbit monoclonal, D8H1X, Cell Signaling), anti-TAZ (rabbit polyclonal, HPA007415, Sigma-Aldrich), anti-PDPN (syrian hamster monoclonal, 127402, Biolegend), anti-CCL19 (goat polyclonal, PA5-47958, Thermo Fisher), anti-CCL21 (goat polyclonal, AF457, R\&D), anti-ER-TR7 (rat monoclonal, sc-73355, Santa Cruz), anti-CD3e (hamster monoclonal, 145-2C11, BD), anti-B220 (rat monoclonal, RA3-6B2, BD), anti-CD31 (hamster monoclonal, 2H8, Millipore), anti-PDGFR $\beta$ (rat monoclonal, APB5, eBioscience), anti-collagen IV (rabbit polyclonal, ab6586, Abcam), anti-Ki-67 (rabbit monoclonal, SP6, Abcam), antiLYVE-1 (rabbit polyclonal, 11-034, Angiobio), anti-caspase-3 (rabbit polyclonal, 9661, Cell Signaling), anti-vimentin (chicken polyclonal, AB5733, Millipore), anticollagen I (rabbit polyclonal, ab34710, Abcam), anti-LT $\beta$ R (rabbit polyclonal, ab70063, Abcam), anti-CD4 (rat monoclonal, GK1.5, BD), anti-CD11b (rat monoclonal, M1/70, BD), anti-CD11c (hamster monoclonal, N418, Bio-Rad), antiperilipin (guinea pig polyclonal, 20R-PP004, Fitzgerald), anti-PNAd (rat monoclonal, MECA-79, BD), anti-ICAM1 (rat monoclonal, YN1/1.7.4, Abcam), anti-Prox1 (rabbit polyclonal, 102-PA32, ReliaTech), and FITC- or Cy3-conjugated anti-aSMA (mouse monoclonal, 1A4, Sigma-Aldrich). FITC-, Cy3- or Cy5conjugated secondary antibodies were purchased from Jackson ImmunoResearch. Lipids were stained with BODIPY (Invitrogen) and nuclei were stained with DAPI (Invitrogen).

Immunological analysis. For adoptive transfer of labeled cells, mononuclear cells were isolated from the spleen and skin-draining LNs from Actb-DsRed and ActbGFP mice. B cells from Actb-DsRed mice and T cells from Actb-GFP mice were further sorted using Dynabeads Untouched Mouse T Cells Kit and Pan B cell Kit (Thermo Fisher). Mixture of $1 \times 10^{7} \mathrm{~T}$ cells and $1 \times 10^{7} \mathrm{~B}$ cells were transferred intravenously into the mouse. LNs of the recipient mice were isolated and analyzed at $24 \mathrm{~h}$ after the adoptive transfer.

For influenza viral infection, mice were immunized by intranasal administration of $1 \times 10^{3} \mathrm{pfu}$ of $\mathrm{A} / \mathrm{PR} / 8$ [A/Peuerto Rico/8/34 (H1N1)]. Then the mice were monitored for weight loss and survival every day for the next 14 days. For detection of influenza-specific antibodies, ELISA plates (Falcon) coated with $20 \mu \mathrm{g} / \mathrm{ml}$ of formalin-inactivated $\mathrm{A} / \mathrm{PR} / 8$ virus in PBS were incubated overnight at $4^{\circ} \mathrm{C}$. Blocking with $1 \%$ BSA in PBS was performed at $37^{\circ} \mathrm{C}$ for $1 \mathrm{~h}$. Serial two-fold dilutions of samples (starting from 1:64 of serum) were applied to plates and incubated for $4 \mathrm{~h}$ at $37^{\circ} \mathrm{C}$. Horseradish peroxidase-conjugated goat anti-mouse IgG antibodies (Southern Biotechnology Associates) were added to each well and incubated for $2 \mathrm{~h}$ at $37^{\circ} \mathrm{C}$. For color development, TMB- $\mathrm{H}_{2} \mathrm{O}_{2}$ solution (Moss) was added as chromogen substrate to each well and incubated for $15 \mathrm{~min}$ at RT. Once stopping solution had been added $(0.5 \mathrm{~N} \mathrm{HCl})$, absorbance was measured at $450 \mathrm{~nm}$ using an ELISA reader (Molecular Devices). Endpoint titers of A/PR/8 virus-specific antibodies were expressed as reciprocal $\log 2$ titers of the highest dilution that showed a level of $>0.1$ absorbance over background.

For intracellular cytokine staining, BD Cytofix/Cytoperm Plus (BD Pharmigen) was used according to the manufacturer's instructions. For the measurement of IFN $\gamma$-producing $\mathrm{CD}^{+} \mathrm{T}$ cells, mononuclear cells were obtained from mediastinal LNs and were incubated with $5 \mathrm{ng} / \mathrm{ml}$ of PMA and with $500 \mathrm{ng} / \mathrm{ml}$ of Ionomycin in the presence of GolgiPlug (BD Pharmingen) for $4 \mathrm{~h}$. The cells were stained with anti-CD8 antibody (rat monoclonal, 53-6.7, BD) and anti-IFN-g antibody (rat monoclonal, XMG1.2, BD), and analyzed with FACS Canto II (BD Biosciences).

For systemic depletion of T cells, anti-mouse CD33 monoclonal Ab $(1 \mathrm{mg} / \mathrm{kg} /$ day for 5 days; Clone 145-2C11, ATCC) was intravenously administered into WT mice. As a control, the same amount of hamster anti-mouse IgG isotype Ab (R\&D Systems) was administered in the same manner.
For analysis of $\mathrm{T}$ cell proliferation in vivo, OVA-specific $\mathrm{T}$ cells were isolated from OT-II mice, labeled with $10 \mu \mathrm{M}$ CFSE, and then adoptively transferred intravenously into the mice. Twenty-four hours after the transfer, $100 \mu \mathrm{g}$ of OVA was injected via footpad. Three days after the injection, mice were euthanatized and mononuclear cells were isolated from popliteal LNs. Activation and CFSE dilution of adaptive transferred OT-II CD4 ${ }^{+}$T cells were analyzed by flow cytometry.

Electron microscopy. LNs were fixed in $2.5 \%$ glutaraldehyde in $0.1 \mathrm{M}$ phosphate buffer ( $\mathrm{pH} 7.4$ ) overnight at $4{ }^{\circ} \mathrm{C}$ and post-fixed with $1 \%$ osmium tetroxide for $2 \mathrm{~h}$ at RT. Samples were dehydrated with series of increasing ethanol concentrations followed by resin embedding. 70-nm-thick ultrathin sections were obtained with an ultramicrotome (UltraCut-UCT, Leica), which were then collected on copper grids. After staining with $2 \%$ uranyl acetate and lead citrate, samples were examined by transmission electron microscope (Tecnai G2 Spirit Twin, FEI) at $120 \mathrm{kV}$.

LN conduit analysis. Conduit staining was achieved by injecting $10 \mu \mathrm{L}$ of saturated FITC (Sigma-Aldrich) in HBSS $(0.1 \mathrm{mg} / \mathrm{mL})$ into the footpad, and the draining popliteal LN was collected at $10 \mathrm{~m}$ after the injection. Samples were fixed in $1 \%$ PFA for $1 \mathrm{~h}$ immediately after the harvest and processed for whole-mount and imaging.

Morphometric analyses. Morphometric analyses of the LNs were performed with images taken from mid-sectioned LNs by photographic analysis using ImageJ software (http://rsb.info.nih.gov/ij) or ZEN 2012 software (Carl Zeiss). Numbers of $\mathrm{Ki}-67^{+}$proliferating or caspase- $3^{+}$apoptotic FRCs, $\mathrm{T}$ cells, and B cells were measured from random four or five $0.045 \mathrm{~mm}^{2}$ fields of LNs and averaged. Nuclear YAP was counted by calculating the proportion of PDGFR $\beta^{+}$FRCs with nuclear YAP from random four or five $0.045 \mathrm{~mm}^{2}$ fields of LNs and averaged. The relative expressions of indicated markers were calculated as $\mathrm{PDPN}^{+}, \mathrm{aSMA}^{+}, \mathrm{PDGFR}^{+}$, collagen $\mathrm{IV}^{+}, \mathrm{CCL}_{1} 9^{+}$or CCL $21^{+}$area divided by Tomato ${ }^{+}, \mathrm{YFP}^{+}$or PDGFR $\beta^{+}$ area. For density measurements, $\mathrm{PDPN}^{+}, \alpha \mathrm{SMA}^{+}, \mathrm{PDGFR}^{+}$, collagen $\mathrm{IV}^{+}$or ER$\mathrm{TR} 7^{+}$area was measured in random four or five $0.045 \mathrm{~mm}^{2}$ area and presented as a percentage of the total measured area. FRC surface area was assessed by employing a $3 \mathrm{D}$ reconstruction analysis using Imaris (Bitplane) ${ }^{46}$. The surface area of Tomato + FRCs was calculated using the single-cell morphometric 'surface' module in Imaris after the $3 \mathrm{D}$ reconstruction of a given image and volume filter was used to reduce background noise. Random four or five $0.045 \mathrm{~mm}^{2}$ fields of LNs were analyzed and individual FRC was distinguished as a separate $3 \mathrm{D}$ surface object by using the 'cutting' tool and DAPI staining was utilized to identify the cell nuclei belonging to each FRC. Percentage of naked conduit and fibril irregularity were measured in up to eight random area of immunofluorescence or TEM images and averaged. Fibril irregularity was assessed by employing a previously described method $^{47}$. Longitudinal section of TEM image was analyzed for non-condensed fibril bundles and unpacked collated fibers with discontinuous, multi-directional pattern were defined as irregular fibrils.

Flow cytometry and cell sorting. Skin-draining LNs (axillary, brachial, cervical, and inguinal LNs) were harvested and cut into small pieces for digestion ${ }^{48}$. Briefly, LNs were digested in $2 \mathrm{ml}$ of enzyme buffer containing $2 \mathrm{mg} / \mathrm{ml}$ collagenase type II (Worthington Biochem), $0.1 \mathrm{mg} / \mathrm{ml}$ DNase (Roche), and $1 \mathrm{mg} / \mathrm{ml}$ dispase (Gibco) at $37^{\circ} \mathrm{C}$ for $30 \mathrm{~min}$. Tissues were gently agitated and pipetted $1-2$ times during digestion to disrupt any cell clumps. When LNs were completely digested, cell suspension was filtered through $40 \mu \mathrm{m}$ nylon cell strainer and washed. Cells were incubated for $20 \mathrm{~min}$ with anti-CD45 Microbeads (Miltenyi). To enrich the stromal cell fraction, hematopoietic cells were depleted using AutoMACS (Miltenyi), according to the manufacturer's instructions. The cells isolated from skin-draining 
a
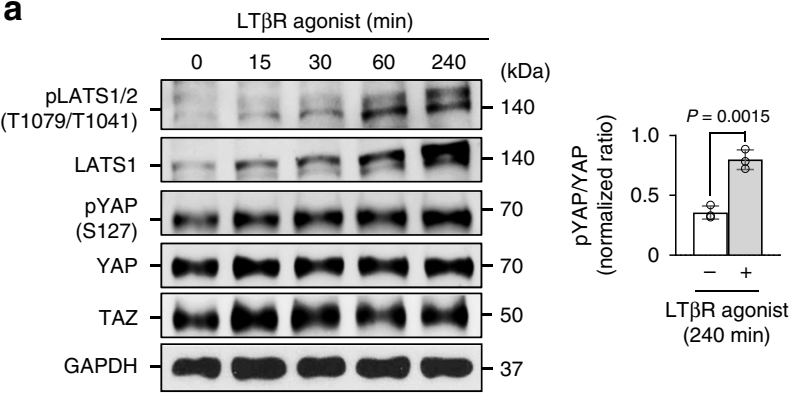

C

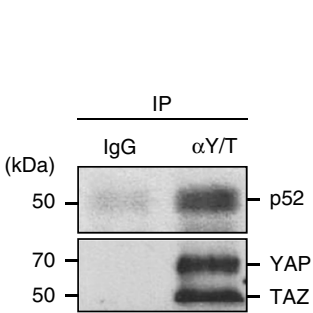

d

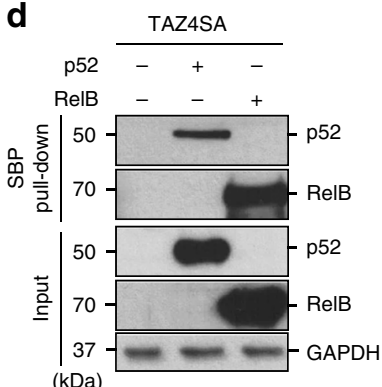

f

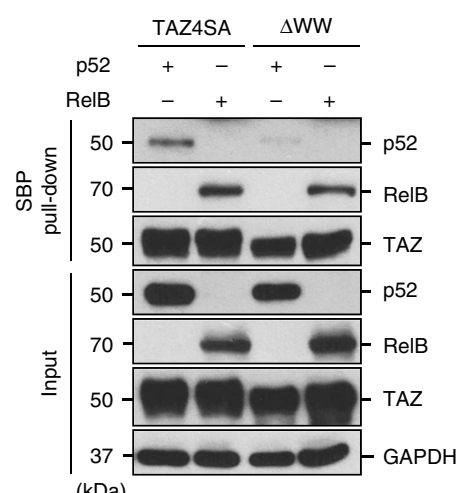

9

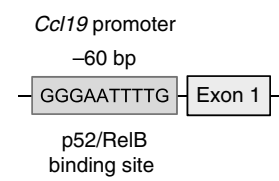

Ccl19-luciferase (WT)

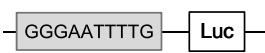

Ccl19-luciferase (Mut) (kDa)

i

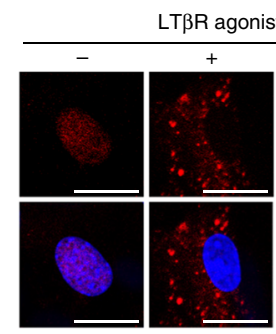

YAP/p52 DAPI

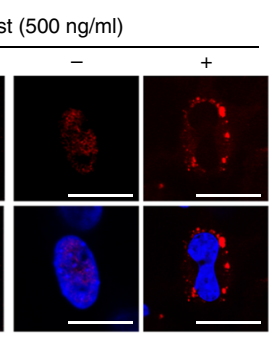

TAZ/p52 DAPI remove cell clumps. After RBC lysis by suspension in ACK lysis buffer for 5 min at $\mathrm{RT}$, the cells were incubated for $30 \mathrm{~min}$ with antibodies (diluted at a ratio of 1:200) in FACS buffer ( $5 \%$ bovine serum in PBS). After several washes, cells were analyzed by FACS Canto II (BD Biosciences) and the acquired data were further evaluated by using FlowJo software (Treestar). Cell sorting was performed with FACS Aria II (Beckton Dickinson). Dead cells were excluded using DAPI staining (Sigma-

Aldrich). The following antibodies were used other than the antibodies described above: anti-CD45 (rat monoclonal, 30-F11, eBioscience), anti-TER-119 (rat monoclonal, TER-119, eBioscience), anti-PDPN (syrian hamster monoclonal, 8.1.1, Biolegend), anti-CD31 (rat monoclonal, MEC 13.3, BD), and anti-CD19 (rat monoclonal, 6D5, Biolegend).

For cell cycle analysis, mice were injected with $1 \mathrm{mg}$ of BrdU solution. Skindraining LNs were harvested at $16 \mathrm{~h}$ after the injection of BrdU and isolated cells were processed with the APC BrdU flow kit (BD Biosciences) according to the manufacturer's protocols. The cell cycle profiles were analyzed with a FACS Canto II (BD Biosciences) and assessed with FlowJo (Treestar).
LNs, spleen, and bone marrow were filtered through a $40 \mu \mathrm{m}$ nylon mesh to
Luc

b
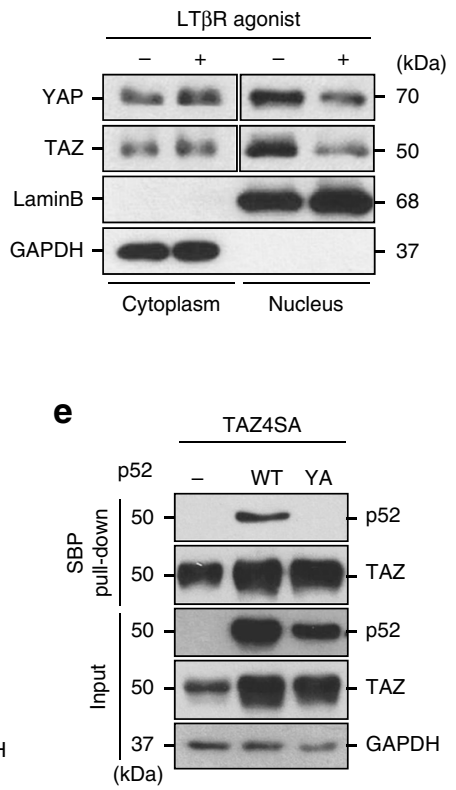

h

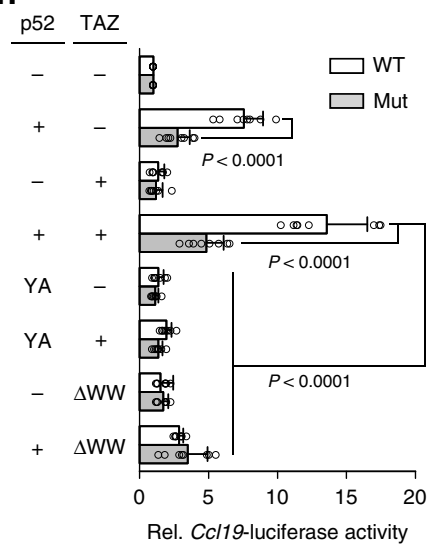

j
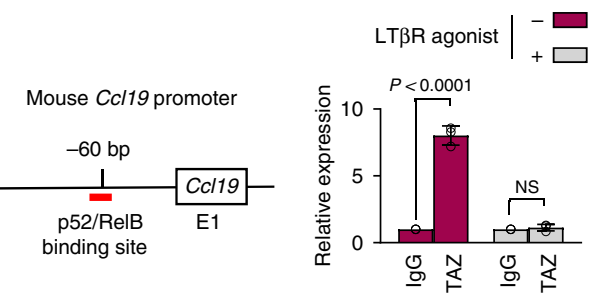

ATAC sequencing. Approximately 20,000 tdTomato $^{+} \mathrm{Lgr}^{+}$stem cells in dermis were FACS-purified and used for ATAC-seq. The ATAC-seq libraries were prepared by employing a previously described method $^{49} .2 \times 101$ paired-end sequencing was performed on Illumina HiSeq-2500. We randomly sampled $2.5 \mathrm{M}$ reads from each sample using samtools view and pooled them into one file so that each sample is equally represented. Peaks were called on the pooled file as discussed in the previous paragraph. We then determined the number of samples overlapping with each master peak using peaks called on individual samples.

Quantitative RT-PCR and RNA sequencing. For quantitative RT-PCR, total RNA was extracted from sorted cells by using Trizol RNA extraction kit (Invitrogen) according to the manufacturer's instructions. Total RNA was reverse transcribed into cDNA using GoScript Reverse Transcription Kit (Promega). Then, quantitative real-time PCR was performed using FastStart SYBR Green Master mix (Roche) and Bio-rad S1000 Thermocycler. GAPDH was used as a reference gene and the 
Fig. 5 YAP/TAZ regulate chemokine expression prior to LT $\beta R$ engagement. a Immunoblot analyses at indicated time points and comparison of normalized pYAP/YAP ratio at $240 \mathrm{~min}$ in cultured FRCs derived from WT mice after stimulation with LT $\beta R$ agonistic antibody ( $500 \mathrm{ng} / \mathrm{ml})$ for indicated time points. b Immunoblot analyses of indicated proteins in nuclear (LaminB) and cytoplasmic (GAPDH) fractions of cultured FRCs after treatment with or without LT $\beta R$ agonistic antibody. c Immunoprecipitation (IP) with anti-lgG or anti-YAP/TAZ ( $\alpha$ Y/T) antibody in primary cultured FRCs derived immunoblot with indicated antibodies. d Pull-down assay with streptavidin resin in HEK-293T cells after transfection with the streptavidin-binding peptide (SBP)-TAZ4SA, with or without plasmids encoding p52 or RelB and immunoblot analysis with indicated antibodies. e Pull-down assay with streptavidin resin in HEK-293T cells after transfection with the (SBP)-TAZ4SA, with or without plasmids encoding p52 (WT) or p52-Y293A mutants (YA) and immunoblot analysis with indicated antibodies. $\mathbf{f}$ Pull-down assay with streptavidin resin in HEK-293T cells after transfection with (SBP)-TAZ4SA or (SBP)-WW domain-deleted TAZ mutant $(\triangle W W$ ) with or without plasmids encoding p52 or RelB and immunoblot analysis with indicated antibodies. $\mathbf{g}$ Diagram depicting the p52/RelB binding site within the mouse Ccl19 promoter and Ccl19 promoter-driven luciferase constructs containing p52/RelB binding site (WT) or the binding site deletion mutant (Mut). $\mathbf{h}$ Comparison of relative luciferase reporter activity using WT and Mut in HEK-293T cells. WT and Mut was co-transfected with or without p52 or p52 mutant (YA) and TAZ or TAZ mutant $(\triangle W W)$ in HEK-293T cells $(n=8)$. $P$ values by one-way ANOVA. $\mathbf{i}$ Representative images of in situ proximity ligation assay showing localizations of YAP or TAZ and p52 after treatment with or without LTRR agonistic antibody in cultured FRCs. Nuclei are stained with DAPI. Scale bars, $50 \mu \mathrm{m}$. j ChIP experiments using IgG or anti-TAZ antibody were performed in MEFs infected with retrovirus encoding CTL or TAZ4SA with or without LT $\beta R$ agonistic antibody. Unless otherwise denoted, similar findings were observed in three independent experiments. Horizontal bars indicate mean \pm SD and $P$ value versus 0 min or Control by two-tailed Student's $t$-test. NS, not significant.

results were presented as relative expressions to control. List of primer sequences are described in Supplementary Table 2.

For RNA sequencing, the construction of library was performed using QuantSeq 3' mRNA-Seq Kit (Lexogen GmbH, Austria) according to the manufacturer's instructions. In brief, each $500 \mathrm{ng}$ total RNA were prepared and an oligo-dT primer containing an Illumina-compatible sequence at its $5^{\prime}$ end was hybridized to the RNA and reverse transcription was performed. After degradation of the RNA template, second strand synthesis was initiated by a random primer containing an Illumina-compatible linker sequence at its $5^{\prime}$ end. The doublestranded library was purified by using magnetic beads to remove all reaction components. The library was amplified to add the complete adapter sequences required for cluster generation. The finished library was purified from PCR components. High-throughput sequencing was performed as single-end 75 sequencing using NextSeq 500 (Illumina). Mapping of RNA-Seq reads were performed using Bowtie2 version 2.1.0, which was used to bring together transcripts, assess their exuberances, and identify DEGs or isoforms using cufflinks. The RT (Read Count) data were processed based on Quantile normalization method using the Genowiz version 4.0.5.6 (Ocimum Biosolutions). The Ingenuity Pathway Analysis tool (QIAGEN) was used to interpret data in the context of canonical pathways, biological processes and networks. Both up- and downregulated identifiers were defined as value parameters for the analysis. Significance of the canonical pathways and biological function and networks were tested by the Fisher Exact test $p$-value, and determined for their activation or inhibition upon activation z-score. For GSEA, gene sets from the Molecular Signatures Database (MSigDB) 5.2 were used for the analysis of the Hippo pathway-responsive transcriptomes. Cluster analysis and heatmap construction were performed using Multiple Experiment Viewer (MeV) from The Institute of Genomic Research (TIGR) and Cluster and TreeView from the Eisen laboratory. Original data are available in the National Center for Biotechnology Information's Gene Expression Omnibus (accession number GSE89742). For presentation of RNA-sequencing data into bar graphs, normalized raw counts $(\log 2)$ were converted by using the following formula: $2^{\text {(x: normalized raw count) }}$. Next, given values were divided by the average value of WT and presented as relative ratio.

Primary culture of FRCs. Skin-draining lymph nodes of indicated mice were harvested, cut into small pieces, and digested ${ }^{48}$. LNs were digested in $2 \mathrm{ml}$ of enzyme buffer containing mixture of $2 \mathrm{mg} / \mathrm{ml}$ collagenase type II (Worthington Biochem), $0.1 \mathrm{mg} / \mathrm{ml}$ DNase (Roche), and $1 \mathrm{mg} / \mathrm{ml}$ dispase (Gibco) at $37^{\circ} \mathrm{C}$ for $30 \mathrm{~min}$. Tissues were gently agitated and pipetted several times during digestion to disrupt any cell clumps. When LNs were completely digested, cell suspension was filtered through $40 \mu \mathrm{m}$ nylon cell strainer and washed. Cells were incubated for 20 min with anti-CD45 Microbeads (Miltenyi). To enrich the stromal cell fraction, hematopoietic cells were depleted using AutoMACS (Miltenyi), according to the manufacturer's instructions. In order to induce FRC differentiation, cells were incubated with DMEM containing $10 \%$ fetal bovine serum and $500 \mathrm{ng} / \mathrm{ml}$ of LT $\beta R$ agonistic antibody (Abcam, Cat. ab65089) for 5 days. For induction of cre activity in primary cultured FRCs derived from Lats $1 / 2^{\mathrm{i} \Delta \mathrm{FRC}}$ or Yap/Taz ${ }^{\Delta \mathrm{FRC}}$ mice, cells were treated with $5 \mu \mathrm{M} 4$-hydroxy-tamoxifen (4-OHT) in $100 \%$ ethanol (EtOH) or $100 \%$ EtOH alone for 2 days as a control. For primary culture of human FRCs, cervical LN specimens were acquired for diagnostic purposes from presently healthy adults with written informed consent according to the protocol approved by the institutional review board of Samsung Medical Center (2018-06-061). LNs were cut into small pieces immediately after the harvest and digested ${ }^{50}$. Following the digestion, cells were covered with media on a tissue culture plate and grown for 4 days to permit fibroblasts to emerge. Culture-expanded monolayer of FRCs under passage 3 that were validated as $\mathrm{CD}_{4}^{-}, \mathrm{CD}^{-} 1^{-}$, and $\mathrm{PDPN}^{+}$were used for the experiments.
For adenoviral infection, human TAZ4SA and YAP5SA cDNAs ${ }^{51}$ were cloned into the pAdtrack-CMV-GFP vector ${ }^{52}$. Cloning vectors were then recombined with the pAdEasy-1 vector in BJ5183-AD-1 electroporation-competent cells (Agilent Technologies). The recombinant DNA was linearized with PacI and introduced into 293AD cells by transfection with Lipofectamine LTX and PLUS reagent (Invitrogen). After verification of GFP expression, cell pellets were centrifuged and resuspended with $10 \%$ glycerol in PBS and lysed with 4 freeze-thaw cycles. Adenoviruses were purified by ultracentrifugation at $46,000 \times g$ for $2 \mathrm{~h}$ at $4{ }^{\circ} \mathrm{C}$ within a discontinuous gradient from 2.2 to $4.0 \mathrm{M} \mathrm{CsCl}$ (Amresco) in $10 \mathrm{mM}$ HEPES (Sigma-Aldrich). The adenovirus-containing layer was removed with a syringe needle, and the viruses were washed twice in a solution containing $10 \mathrm{mM}$ Tris- $\mathrm{HCl}(\mathrm{pH} 8.0)$ and $2 \mathrm{mM} \mathrm{MgCl}_{2}$ using an Amicon Ultra Centrifugal Filter (Sigma-Aldrich). Virus titration was performed by counting exposed 293AD or target cells positive for GFP with a fluorescence microscope.

Immunoblotting. For immunoblot analysis, cells were lysed on ice in RIPA lysis buffer supplemented with protease and phosphatase inhibitors (Roche). Cell lysates were centrifuged for $10 \mathrm{~min}$ at $4{ }^{\circ} \mathrm{C}, 13,000 \mathrm{rpm}$. Protein concentrations of the supernatants were quantitated using the detergent-insensitive Pierce BCA protein assay kit (Thermo Fischer, 23227). Laemmli's buffer was added to total protein lysates and samples were denatured at $95^{\circ} \mathrm{C}$ for $5 \mathrm{~min}$. Aliquots of each protein lysate $(10-20 \mu \mathrm{g})$ were subjected to SDS polyacrylamide gel electrophoresis. After electrophoresis, proteins were transferred to nitrocellulose membranes and blocked for 30 min with $5 \%$ skim milk in TBST ( $0.1 \%$ Tween 20 in TBS). For phosphorylated protein detection, membranes were blocked with $2 \%$ BSA in TBS. For detection of protein stability, protein synthesis was blocked by treatment of $50 \mu \mathrm{g} / \mathrm{ml}$ cycloheximide (CHX) for indicated time. Primary antibodies (diluted at a ratio of 1:1000 in blocking solution) were incubated overnight at $4{ }^{\circ} \mathrm{C}$. After washes, membranes were incubated with anti-rabbit (CST, \#7074) or anti-mouse (CST, \#7076) secondary peroxidase coupled antibody (diluted at a ratio of 1:5000 in TBST) for $1 \mathrm{~h}$ at RT. Target proteins were detected using ECL western blot detection solution (Millipore, WBKLS0500). The following antibodies were used for immunoblotting: anti-YAP (rabbit monoclonal, D8H1X, Cell Signaling), antiphospho-YAP (rabbit polyclonal, 4911, Cell Signaling), anti-YAP/TAZ (rabbit monoclonal, D24E4, Cell Signaling), anti-TAZ (rabbit monoclonal, V386, Cell Signaling), anti-LATS1 (rabbit monoclonal, C66B5, Cell Signaling), anti-phosphoLATS1/2 (rabbit monoclonal, D57D3, Cell Signaling), anti-CTGF (rabbit polyclonal, ab6992, Abcam), anti-RelB (rabbit monoclonal, D7D7W, Cell Signaling), anti-CTGF (rabbit polyclonal, ab6992, Abcam), anti-p100/p52 (rabbit polyclonal, 4882, Cell Signaling), anti-p100/p52 (rabbit monoclonal, sc-7386, Santa Cruz), anti-NIK (rabbit polyclonal, 4994, Cell Signaling), anti-LaminB (rabbit monoclonal, D4Q4Z, Cell Signaling), anti-GAPDH (rabbit monoclonal, D16H11, Cell Signaling), and anti- $\beta$ actin (rabbit monoclonal, AC-74, Sigma-Aldrich).

For nuclear-cytoplasmic fractionation of cells, harvested cells were resuspended in hypotonic lysis buffer (10 mM HEPES [pH 7.8], $10 \mathrm{mM} \mathrm{KCl,} 1.5 \mathrm{mM} \mathrm{MgCl}_{2}$, $0.5 \mathrm{mM}$ DTT, and protease inhibitors). To break plasma membrane, the resuspended cells were mixed with $0.3 \% \mathrm{NP}-40$ by vortexing for $5 \mathrm{~s}$, and the cytoplasmic fraction was obtained from the supernatant after centrifugation. After several washes with PBS, the pellet was boiled in Laemmli sample buffer and used as the nuclear fraction. The uncropped and unprocessed scans with marker positions of all blots were included in the Source Data file.

Pull-down assay and co-immunoprecipitation. The N-terminal Flag-SBP tagged human TAZ4SA cDNAs were cloned into the pcDNA3.1 vector (Thermo Fischer, Cat. V790-20). Flag-p52 and Flag-RelB were purchased from Addgene (plasmid \# 20019, \# 20017). Each mutant construct (TAZ4SA $\triangle$ W, p52 YA and RelB YA) was generated by overlap extension PCR. HEK293T cells were cultured in 6-well plate 
a

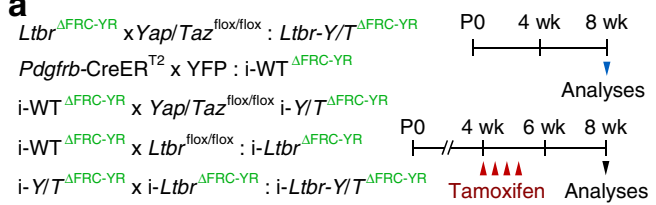

b
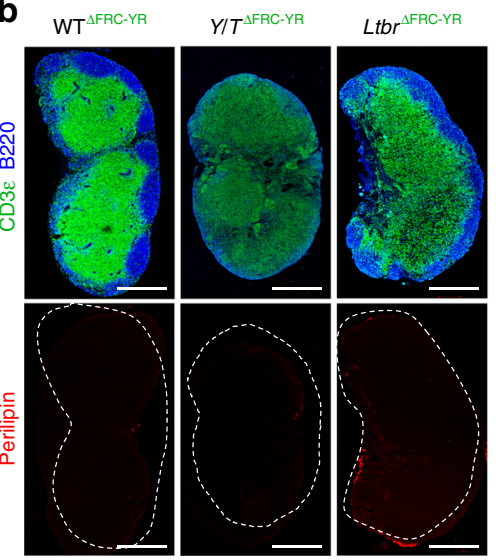

$L$ tbr-YIT $T^{\triangle F R C-Y R}$
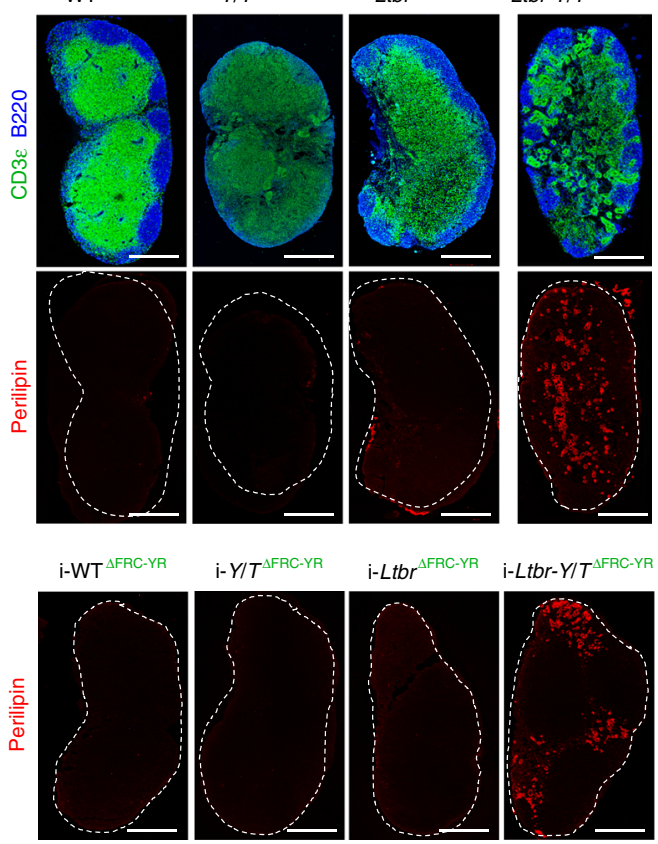

C

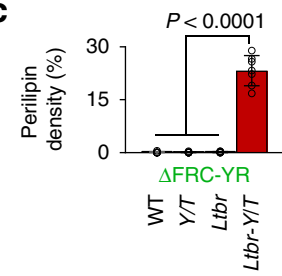

d

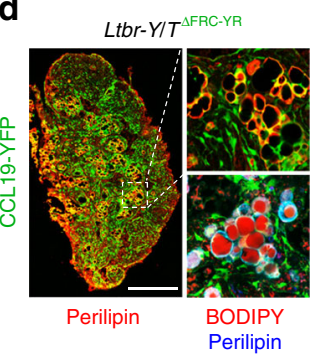

$\mathbf{f}$

Primary culture of FRCs
from i-Ltbr-Y/T $T^{\Delta F R C-Y R}$ mice

$\begin{array}{llll}0 & 4 & 5 & 6(d)\end{array}$

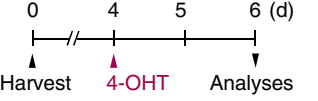

e

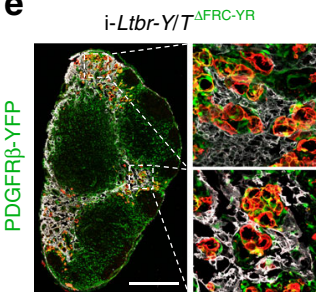

Perilipin LYVE-1

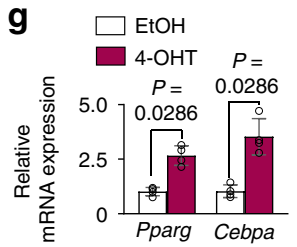

h

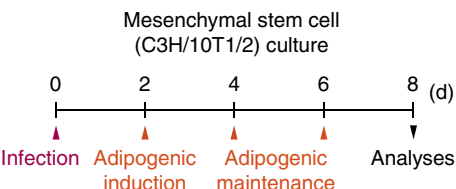

i

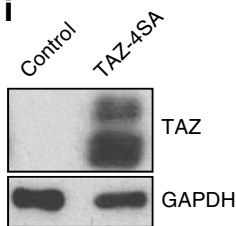

j

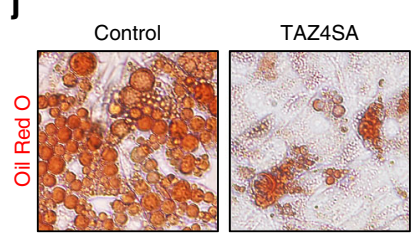

$\mathbf{k}$

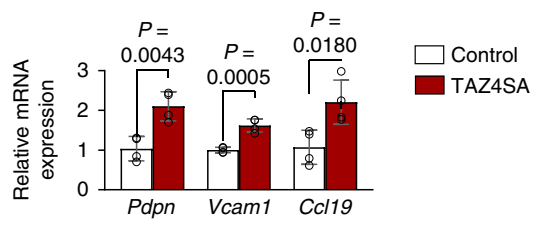

I

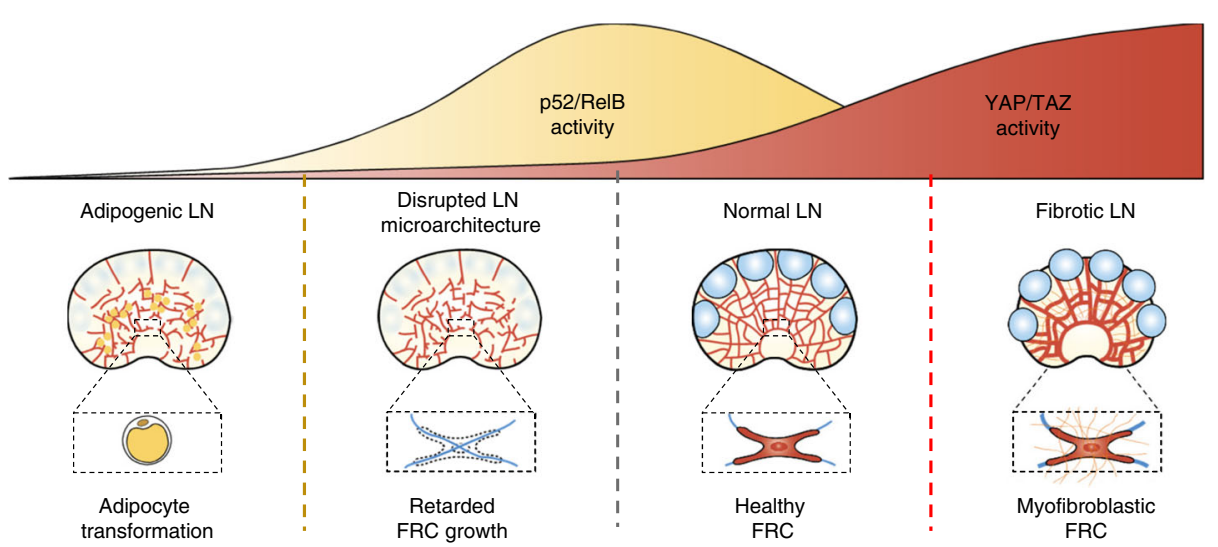

and were co-transfected with indicated constructs using polyethylenimine Max (Polyscience, Cat. 24765-1). Two days after transfection, the cells were harvested, lysed with NETN buffer (20 mM Tris-HCl (pH 7.4), $100 \mathrm{mM} \mathrm{NaCl}, 1 \mathrm{mM}$ EDTA, $0.5 \%$ Nonidet P- 40 , and protease inhibitors). Cell extracts $(1 \mathrm{mg})$ were incubated with Streptavidin Agarose (Pierce, Cat. 20359) for $2 \mathrm{~h}$ at $4{ }^{\circ} \mathrm{C}$, and then the beads were washed three times with lysis buffer and boiled with Laemmli sample buffer. For co-immunoprecipitation, harvested FRCs were lysed with NETN buffer. Cell extracts $\left(0.5 \mathrm{mg}\right.$ ) were incubated overnight at $4^{\circ} \mathrm{C}$ with $0.5 \mu \mathrm{g}$ of anti-IgG (rabbit polyclonal, H-270, Santa Cruz) or anti-YAP/TAZ (rabbit monoclonal, D24E4, Cell Signaling) antibody. The extracts were incubated with $20 \mu \mathrm{l}$ of protein A/G agarose beads (Pierce) for $1 \mathrm{~h}$, and then the beads were washed three times with lysis buffer
(Triton X-100 reduced to $0.1 \%$ ) and boiled with Laemmli sample buffer. For detection of p52, the TrueBlot anti-Rabbit IgG HRP (ROCKLAND, Cat. 18-881631) was used as secondary antibody for reducing the heavy chain interference.

Luciferase assay. The indicated portion of the $C c l 19$ genomic locus including p52 binding site was cloned into the pGL3-Basic vector (Promega, Cat. E1751). Mutant construct was generated with deletion of $\mathrm{p} 52$ binding site by overlap extension PCR. HEK293T cells were cultured in 24-well plate and were co-transfected with a 100 ng HA-TAZ4SA (WT or $\triangle \mathrm{W}$ ), 100 ng Flag-p52 (WT or YA), 200 ng CCL19 luciferase (WT or Mut) or $20 \mathrm{ng}$ CMV-Renilla per well by using polyethylenimine 
Fig. 6 Depletion of Yap/Taz transforms mesenchymal FRC precursors into adipocytes. a Diagram for analyses of indicated mice at 8-weeks old with or without tamoxifen delivery from 4-weeks old. b, c Representative images and comparisons of perilipin ${ }^{+}$adipocytes within the inguinal LN (dashed line) in indicated mice $(n=7)$. Scale bars, $400 \mu \mathrm{m}$. $\mathbf{d}$ Representative images of inguinal LN filled with adipocytes in $L t b r-Y / T \Delta F R C-Y R$ mice $(n=6)$. Right upper panel shows the magnified view of the region within the white dashed box and yellow arrowheads in the right lower panel indicate CCL19-YFP+perilipin ${ }^{+}$BODIPY ${ }^{+}$adipocytes. Scale bars, $500 \mu \mathrm{m}$ (left panel); $100 \mu \mathrm{m}$ (right lower panel). e Representative images of perilipin ${ }^{+}$adipocytes along the LYVE-1+ lymphatic vessels (dashed-boxes) in inguinal LN of $\mathrm{i}-\mathrm{Ltbr}-Y / T \Delta F R C-Y R$ mice $(n=6)$. Scale bar, $400 \mu \mathrm{m}$. f, Diagram for primary culture of FRCs derived from $\mathrm{i}-\mathrm{Ltbr}-\mathrm{Y} / T^{\Delta F R C-Y R}$ mice for 4 days and treatment with $\mathrm{EtOH}$ or $4-\mathrm{OHT}$ for their analyses at 2 days after the treatment. $\mathbf{g}$ Comparisons of indicated mRNA expression normalized to Gapdh in primary cultured FRCs after treatment with EtOH or 4-OHT for 2 days $(n=4)$. $\mathbf{h}$ Diagram for adipogenic culture of mesenchymal stem cells (C3H/10T1/2) infected with an adenovirus to induce overexpression of active TAZ (TAZ4SA) for their analyses at 8 days after the infection. i Immunoblot analyses of indicated proteins in mesenchymal stem cells (C3H/10T1/2) infected with an adenovirus to induce overexpression of active TAZ (TAZ4SA) or control. $\mathbf{j}$ Representative images of Oil Red O staining in mesenchymal stem cells (C3H/10T1/2) induced with adipogenic cocktail after infected with an adenovirus to induce overexpression of active TAZ (TAZ4SA). $\mathbf{k}$ Comparisons of indicated mRNA expression normalized to Gapdh in mesenchymal stem cells $(\mathrm{C} 3 \mathrm{H} / 10 \mathrm{~T} 1 / 2)$ infected with an adenovirus to induce overexpression of active TAZ (TAZ4SA) for their analyses at 2 days after the infection $(n=4)$. I Schematic images proposing the importance of coordination of YAP/TAZ activity and LT $\beta R$ coupling in FRCs during LN growth and maintenance. Unless otherwise denoted, horizontal bars indicate mean \pm SD and $P$ values versus non-Y/T $\triangle F R C-Y R$ or non-i-Ltbr $\Delta F R C-Y R$ or EtOH or Control by two-tailed Mann-Whitney $U$ test.

Max. Twenty-four hours later, cells were harvested, lysed, and assayed with the Dual Luciferase Reporter Assay System (Promega, Cat. E1960). List of primer sequences for luciferase assay are described in Supplementary Table 2.

ChIP-qPCR analysis. MEF cells were fixed with $1 \%$ formaldehyde for $10 \mathrm{~min}$ and then neutralized with $125 \mathrm{mM}$ glycine for $5 \mathrm{~min}$ at RT. The cells were washed with PBS and then lysed with ChIP dilution buffer [50 mM HEPES (pH 7.5), $155 \mathrm{mM}$ $\mathrm{NaCl}, 1 \%$ Triton X-100, $0.1 \%$ sodium deoxycholate, $123 \mathrm{mM}$ EDTA] containing $1 \%$ SDS. The DNA in the cell lysates was fragmented by sonication using a bioruptor sonicator. The cell lysates were centrifuged at $13,000 \mathrm{rpm}$ for $15 \mathrm{~min}$ at $4{ }^{\circ} \mathrm{C}$, and the resulting supernatants were further diluted with ChIP dilution buffer. They were then incubated overnight at $4{ }^{\circ} \mathrm{C}$ with either TAZ antibody (Sigma,

HPA007415) or IgG (Santa Cruz Biotechnology). The next day, protein A/G beads (Gendepot) were added and the samples were incubated for an additional $3 \mathrm{~h}$ at $4{ }^{\circ} \mathrm{C}$. The beads were then isolated with centrifugation, washed with ChIP wash buffer [10 mM Tris- $\mathrm{HCl}(\mathrm{pH} 8.0), 250 \mathrm{mM} \mathrm{LiCl}, 0.5 \%$ Nonidet P-40, $0.5 \%$ sodium deoxycholate, $1 \mathrm{mM}$ EDTA], and suspended in SDS lysis buffer [ $50 \mathrm{mM}$ Tris-HCl (pH 8.0), $10 \mathrm{mM}$ EDTA, $1 \%$ SDS] for overnight incubation at $65^{\circ} \mathrm{C}$. The beads were then removed, and the remaining material was incubated for $2 \mathrm{~h}$ at $55^{\circ} \mathrm{C}$ with proteinase $\mathrm{K}(20 \mathrm{mg} / \mathrm{ml})$ and glycogen $(20 \mathrm{mg} / \mathrm{ml})$. After a final $1 \mathrm{~h}$ incubation with RNaseA at $37^{\circ} \mathrm{C}$, the DNA was purified using standard procedures and analyzed for "GGGRNNYYCC" motif. List of primer sequences for ChIP-qPCR are described in Supplementary Table 2

In situ proximity ligation assay. Cells cultured on confocal dishes were fixed with $4 \%$ paraformaldehyde for $20 \mathrm{~min}$ at RT, permeabilized and incubated with primary antibodies at $4{ }^{\circ} \mathrm{C}$. For in situ proximity ligation assay, protein-protein interactions between YAP or TAZ and p52 were detected with secondary proximity probes (Anti-Rabbit Plus and Anti-Mouse Minus) according to the Duolink in situ Fluorescence User Guide (Sigma-Aldrich).

Adipogenic induction. In order to induce adipogenic differentiation, confluent cells were incubated with adipogenic differentiation medium [DMEM containing $10 \%$ fetal bovine serum, $5 \mu \mathrm{g} / \mathrm{ml}$ insulin (Sigma-Aldrich), $0.5 \mathrm{mM} \mathrm{3-isobutyl-1-}$ methylxanthine (IBMX, Sigma-Aldrich) and $1 \mu \mathrm{M}$ dexamethasone (SigmaAldrich)]. After 3 days, adipogenic differentiation medium was replaced with maintenance medium (DMEM containing 10\% fetal bovine serum and $5 \mu \mathrm{g} / \mathrm{ml}$ insulin). For Oil Red O staining, cells were fixed with $4 \%$ paraformaldehyde for $40 \mathrm{~min}$ at RT. After being washed with PBS and $60 \%$ isopropanol, cells were incubated with filtered Oil Red O working solution for 50 min at RT. After staining, several washes with PBS and $60 \%$ isopropanol were performed to reduce nonspecific staining. Images of stained cells were captured by a microscope equipped with a CCD camera (Carl Zeiss).

Statistical analyses. All values are presented as mean \pm standard deviation (SD). For continuous data, statistical significance was determined by the Mann-Whitney $U$ test or Student's $t$-test between two groups and one-way ANOVA for multiplegroup comparison. The survival curve was evaluated using the Kaplan-Meier method, and the statistical difference was analyzed using the log-rank test. Statistical analysis was performed with GraphPad Prism. Statistical significance was set to $P<0.05$.

Reporting summary. Further information on research design is available in the Nature Research Reporting Summary linked to this article.

\section{Data availability}

The RNA-sequencing and ATAC-sequencing data generated with this study have been deposited in Gene Expression Omnibus under the accession number GSE89742. The source data underlying all Figs. and Supplementary Figs. are provided as a Source Data file. A Reporting summary for this article is available as a Supplementary Information file. All other data that support the findings of this study are available from the corresponding author upon reasonable request.

Received: 20 June 2019; Accepted: 31 December 2019; Published online: 24 January 2020

\section{References}

1. Fletcher, A. L., Acton, S. E. \& Knoblich, K. Lymph node fibroblastic reticular cells in health and disease. Nat. Rev. Immunol. 15, 350-361 (2015).

2. Petrova, T. V. \& Koh, G. Y. Organ-specific lymphatic vasculature: from development to pathophysiology. J. Exp. Med. 215, 35-49 (2018).

3. Chang, J. E. \& Turley, S. J. Stromal infrastructure of the lymph node and coordination of immunity. Trends Immunol. 36, 30-39 (2015).

4. Bajenoff, M., Glaichenhaus, N. \& Germain, R. N. Fibroblastic reticular cells guide T lymphocyte entry into and migration within the splenic T cell zone. $J$. Immunol. 181, 3947-3954 (2008).

5. Siegert, S. \& Luther, S. A. Positive and negative regulation of T cell responses by fibroblastic reticular cells within paracortical regions of lymph nodes. Front Immunol. 3, 285 (2012).

6. Cremasco, V. et al. B cell homeostasis and follicle confines are governed by fibroblastic reticular cells. Nat. Immunol. 15, 973-981 (2014).

7. Riedel, A., Shorthouse, D., Haas, L., Hall, B. A. \& Shields, J. Tumor-induced stromal reprogramming drives lymph node transformation. Nat. Immunol. 17, $1118-1127$ (2016)

8. Tamura, N. et al. Tumor histology in lymph vessels and lymph nodes for the accurate prediction of outcome among breast cancer patients treated with neoadjuvant chemotherapy. Cancer Sci. 100, 1823-1833 (2009).

9. Estes, J. D. Pathobiology of HIV/SIV-associated changes in secondary lymphoid tissues. Immunol. Rev. 254, 65-77 (2013).

10. Zeng, M. et al. Cumulative mechanisms of lymphoid tissue fibrosis and $\mathrm{T}$ cell depletion in HIV-1 and SIV infections. J. Clin. Invest. 121, 998-1008 (2011).

11. Lu, T. T. \& Browning, J. L. Role of the lymphotoxin/LIGHT system in the development and maintenance of reticular networks and vasculature in lymphoid tissues. Front Immunol. 5, 47 (2014).

12. Benezech, C. et al. Lymphotoxin-beta receptor signaling through NFkappaB2-RelB pathway reprograms adipocyte precursors as lymph node stromal cells. Immunity 37, 721-734 (2012).

13. Buckley, C. D., Barone, F., Nayar, S., Benezech, C. \& Caamano, J. Stromal cells in chronic inflammation and tertiary lymphoid organ formation. Аnnu Rev. Immunol. 33, 715-745 (2015).

14. Barone, F. et al. Stromal fibroblasts in tertiary lymphoid structures: a novel target in chronic inflammation. Front Immunol. 7, 477 (2016).

15. Majumder, S. et al. IL-17 metabolically reprograms activated fibroblastic reticular cells for proliferation and survival. Nat. Immunol. 20, 534-545 (2019).

16. van de Pavert, S. A. et al. Chemokine CXCL13 is essential for lymph node initiation and is induced by retinoic acid and neuronal stimulation. Nat. Immunol. 10, 1193-1199 (2009). 
17. Kong, Y. Y. et al. OPGL is a key regulator of osteoclastogenesis, lymphocyte development and lymph-node organogenesis. Nature 397, 315-323 (1999).

18. Kim, D. et al. Regulation of peripheral lymph node genesis by the tumor necrosis factor family member TRANCE. J. Exp. Med. 192, 1467-1478 (2000)

19. Benezech, C. et al. Ontogeny of stromal organizer cells during lymph node development. J. Immunol. 184, 4521-4530 (2010).

20. Chai, Q. et al. Maturation of lymph node fibroblastic reticular cells from myofibroblastic precursors is critical for antiviral immunity. Immunity 38, 1013-1024 (2013).

21. Onder, L. et al. Lymphatic endothelial cells control initiation of lymph node organogenesis. Immunity 47, 80-92 e84 (2017).

22. Yu, F. X., Zhao, B. \& Guan, K. L. Hippo pathway in organ size control, tissue homeostasis, and cancer. Cell 163, 811-828 (2015).

23. Halder, G., Dupont, S. \& Piccolo, S. Transduction of mechanical and cytoskeletal cues by YAP and TAZ. Nat. Rev. Mol. Cell Biol. 13, 591-600 (2012).

24. Irvine, K. D. \& Harvey, K. F. Control of organ growth by patterning and hippo signaling in Drosophila. Cold Spring Harb. Perspect. Biol. 7, a019224 (2015).

25. Piccolo, S., Dupont, S. \& Cordenonsi, M. The biology of YAP/TAZ: hippo signaling and beyond. Physiol. Rev. 94, 1287-1312 (2014).

26. Cho, H. et al. YAP and TAZ negatively regulate Proxl during developmental and pathologic lymphangiogenesis. Circ. Res. 124, 225-242 (2019).

27. Xin, M. et al. Regulation of insulin-like growth factor signaling by Yap governs cardiomyocyte proliferation and embryonic heart size. Sci. Signal 4, ra70 (2011).

28. Xin, M. et al. Hippo pathway effector Yap promotes cardiac regeneration. Proc. Natl Acad. Sci. USA 110, 13839-13844 (2013).

29. Heallen, T. et al. Hippo pathway inhibits Wnt signaling to restrain cardiomyocyte proliferation and heart size. Science 332, 458-461 (2011).

30. Kim, M. et al. cAMP/PKA signalling reinforces the LATS-YAP pathway to fully suppress YAP in response to actin cytoskeletal changes. EMBO J. 32, 1543-1555 (2013).

31. Hiruma, K., Hirsch, R., Patchen, M., Bluestone, J. A. \& Gress, R. E. Effects of anti-CD3 monoclonal antibody on engraftment of T-cell-depleted bone marrow allografts in mice: host T-cell suppression, growth factors, and space. Blood 79, 3050-3058 (1992).

32. Loubaki, L., Tremblay, T. \& Bazin, R. In vivo depletion of leukocytes and platelets following injection of $\mathrm{T}$ cell-specific antibodies into mice. J. Immunol. Methods 393, 38-44 (2013).

33. Xiao, Y. et al. Hippo signaling plays an essential role in cell state transitions during cardiac fibroblast development. Dev. Cell 45, 153-169 e156 (2018).

34. dos Santos, G. et al. Vimentin regulates activation of the NLRP3 inflammasome. Nat. Commun. 6, 6574 (2015).

35. Cildir, G., Low, K. C. \& Tergaonkar, V. Noncanonical NF-kappaB signaling in health and disease. Trends Mol. Med. 22, 414-429 (2016).

36. Vallabhapurapu, S. \& Karin, M. Regulation and function of NF-kappaB transcription factors in the immune system. Annu Rev. Immunol. 27, 693-733 (2009).

37. Varelas, X. The Hippo pathway effectors TAZ and YAP in development, homeostasis and disease. Development 141, 1614-1626 (2014).

38. Barrallo-Gimeno, A. \& Nieto, M. A. Evolutionary history of the Snail/Scratch superfamily. Trends Genet. 25, 248-252 (2009).

39. Tang, Y., Feinberg, T., Keller, E. T., Li, X. Y. \& Weiss, S. J. Snail/Slug binding interactions with YAP/TAZ control skeletal stem cell self-renewal and differentiation. Nat. Cell Biol. 18, 917-929 (2016).

40. Cristancho, A. G. \& Lazar, M. A. Forming functional fat: a growing understanding of adipocyte differentiation. Nat. Rev. Mol. Cell Biol. 12, 722-734 (2011).

41. White, A. et al. Lymphotoxin a-dependent and -independent signals regulate stromal organizer cell homeostasis during lymph node organogenesis. Blood 110, 1950-1959 (2007)

42. Hong, J. H. et al. TAZ, a transcriptional modulator of mesenchymal stem cell differentiation. Science 309, 1074-1078 (2005).

43. Cheng, $\mathrm{H}$. W. et al. Origin and differentiation trajectories of fibroblastic reticular cells in the splenic white pulp. Nat. Commun. 10, 1739 (2019).

44. Youn, D. Y. et al. Bis deficiency results in early lethality with metabolic deterioration and involution of spleen and thymus. Am. J. Physiol. Endocrinol. Metab. 295, E1349-E1357 (2008).

45. Sheikh, A. Q., Misra, A., Rosas, I. O., Adams, R. H. \& Greif, D. M. Smooth muscle cell progenitors are primed to muscularize in pulmonary hypertension. Sci. Transl. Med. 7, 308ra159 (2015).

46. Novkovic, M. et al. Topological small-world organization of the fibroblastic reticular cell network determines lymph node functionality. PLoS Biol. 14, e1002515 (2016).
47. Drumea-Mirancea, M. et al. Characterization of a conduit system containing laminin-5 in the human thymus: a potential transport system for small molecules. J. Cell Sci. 119, 1396-1405 (2006).

48. Fletcher, A. L. et al. Lymph node fibroblastic reticular cells directly present peripheral tissue antigen under steady-state and inflammatory conditions. $J$. Exp. Med. 207, 689-697 (2010).

49. Buenrostro, J. D., Giresi, P. G., Zaba, L. C., Chang, H. Y. \& Greenleaf, W. J. Transposition of native chromatin for fast and sensitive epigenomic profiling of open chromatin, DNA-binding proteins and nucleosome position. Nat. Methods 10, 1213-1218 (2013).

50. Knoblich, K. et al. The human lymph node microenvironment unilaterally regulates T-cell activation and differentiation. PLoS Biol. 16, e2005046 (2018).

51. Jeong, S. H. et al. Hippo-mediated suppression of IRS2/AKT signaling prevents hepatic steatosis and liver cancer. J. Clin. Investig. 128, 1010-1025 (2018).

52. He, T. C. et al. A simplified system for generating recombinant adenoviruses. Proc. Natl Acad. Sci. USA 95, 2509-2514 (1998).

\section{Acknowledgements}

We thank Hyun Tae Kim for technical assistance. S.Y.C. (2018R1D1A1B07044974) and H.B. (NRF- 2014-Fostering Core Leaders of the Future Basic Science Program/Global Ph. D. Fellowship Program Grant) are supported by the National Research Foundation of Korea (NRF) grant funded by the Korean government. This study was supported by the Institute for Basic Science (IBS-R025-D1, G.Y.K.) funded by the Ministry of Science, ICT and Future Planning, Korea and the Human Frontiers Science Program (RGP0034/2016, B.L. and G.Y.K.).

\section{Author contributions}

S.Y.C., H.B., and S.H.J. designed and performed the experiments, analyzed and interpreted the data; S.Y.C., H.B., S.H.J., I.P., H.C., and G.Y.K. wrote and edited the paper; SPH contributed to in vitro experiments; D.H.L. and M.J.Y. performed ATAC sequencing; C.K.L. and S.H.S. contributed to in vivo experiments; J.S.P. and J.C. participated in manuscript preparation; J.Y.J., J.H.M., H.S.J., and J.M.K. provided human samples; L.O R.A., and B.L. provided the mice and critical comments on this study; J.H.S. performed immunological experiments; and D.S.L. and G.Y.K. directed and supervised the project.

\section{Competing interests}

The authors declare no competing interests.

\section{Additional information}

Supplementary information is available for this paper at https://doi.org/10.1038/s41467 020-14293-1.

Correspondence and requests for materials should be addressed to D.-S.L. or G.Y.K

Peer review information Nature Communications thanks the anonymous reviewer(s) for their contribution to the peer review of this work.

Reprints and permission information is available at http://www.nature.com/reprints

Publisher's note Springer Nature remains neutral with regard to jurisdictional claims in published maps and institutional affiliations.

Open Access This article is licensed under a Creative Commons Attribution 4.0 International License, which permits use, sharing, adaptation, distribution and reproduction in any medium or format, as long as you give appropriate credit to the original author(s) and the source, provide a link to the Creative Commons license, and indicate if changes were made. The images or other third party material in this article are included in the article's Creative Commons license, unless indicated otherwise in a credit line to the material. If material is not included in the article's Creative Commons license and your intended use is not permitted by statutory regulation or exceeds the permitted use, you will need to obtain permission directly from the copyright holder. To view a copy of this license, visit http://creativecommons.org/ licenses/by/4.0/

(c) The Author(s) 2020 